\title{
Chapter 15 \\ The Role of Intrinsic Cell Properties in Synchrony of Neurons Interacting via Electrical Synapses
}

\author{
David Hansel, Germán Mato, and Benjamin Pfeuty
}

\begin{abstract}
The study of the synchronization of neural activity has to deal with the existence of a great diversity of neuronal and synaptic properties, which both may influence the collective behavior of neurons. In this chapter, we present an approach that allows to disentangle the respective role of neuronal and synaptic properties in synchronization. It relies on the theory of weakly coupled oscillators and on the concept of infinitesimal Phase Response Curve (iPRC) which is relevant for systems where neurons fire periodically. Applying this theory to networks of neurons coupled with electrical synapses provides a unifying framework explaining how their synchronization behavior depends on the shape of the iPRC. It reveals that synchronization mediated by electrical synapses is more versatile than previously considered. It depends on the intrinsic currents and morphology of neurons as well as on their combination with inhibitory synapses.
\end{abstract}

D. Hansel ( $\square)$

Neurophysique et Physiologie, CNRS UMR8119, Université Paris Descartes, 45 rue des Saint-Pères 75270 Paris, France

e-mail: david.hansel@univ-paris5.fr

G. Mato

Centro Atómico Bariloche and Instituto Balseiro (CNEA and CONICET),

8400 San Carlos de Bariloche, Argentina

e-mail: matog@cab.cnea.gov.ar

B. Pfeuty

PhLAM, CNRS UMR8523, Université Lille I, F-59655 Villeneuve d'Ascq, France

e-mail: pfeuty@phlam.univ-lille1.fr 


\section{Introduction}

Synchronous electrical activity in the nervous system is known since the discovery of electroencephalography in the 1930s (Berger 1929) and the first studies on the $\alpha$ rhythm in mammals. Following these pioneering works, many other examples of synchronous activity have been discovered in various species and areas of the nervous system. They differ greatly in the power spectrum and the spatiotemporal patterns of the activity as well as in the conditions of occurrence, which can be pathological or physiological. The tremendous progress in experimental techniques which has occurred in the last 20 years, has opened new horizons to establish the relationship between synchronous electrical activities and cellular properties.

The observed synchronous patterns of activity in the nervous system raise several theoretical issues regarding the relationship between the structure of activity patterns and the cellular properties of the neurons, the nature of their interactions, the local and global architectures of the networks, and their interconnections with other brain areas. Furthermore, understanding how noise, heterogeneities, and spatial fluctuations of connectivity patterns affect synchrony is a fundamental question for neuroscientists, and more specifically for theoreticians. A suitable framework to address these issues is the physics of extended dynamical systems. One possible approach is to study the cooperative dynamical states in simplified network models, which within some limits can allow in-depth analytical investigations based on statistical mechanics of pure and disordered systems, the theory of stochastic systems and the physics of non-linear dynamical systems. Subsequently, one extends the analytical investigations by numerical simulations beyond the limits which can be treated analytically. This approach has lead to substantial progress in our understanding of general mechanisms of neuronal synchrony.

Electrical synapses have long been known to exist in invertebrates (Watanabe 1958; Furshpan and Potter 1959) but it is only recently that evidence of their ubiquity has been unequivocally found in the mammalian brain. Remarkably, electrical synapses in the central nervous system are frequently found to connect GABAergic interneurons. This is, for instance, the case in the striatum (Kita et al. 1990), the hippocampus (Venence et al. 2000), the cerebellum (MannMetzer and Yarom 1999), the reticular thalamic nucleus (Landisman et al. 2002), or in the neocortex (Gibson et al. 1999; Galarreta and Hestrin 1999; Fukuda and Kosaka 2000). In the neocortex, electrical as well as inhibitory synapses exist between Fast-Spiking (FS) interneurons (Gibson et al. 1999; Galarreta and Hestrin 2001) or between Multipolar Bursting neurons (Blatow et al. 2003). In contrast, low-threshold-spiking (LTS) interneurons interact mostly via electrical synapses, the inhibitory synapses between them being sparse (Gibson et al. 1999; Amitai et al. 2002). In the FS population, cells also interact via inhibition. FS and LTS neurons interact conversely via inhibition but not via electrical synapses.

Recent experiments have shown that electrical synapses may be involved in synchronizing neural activity. Blocking of inhibition and excitation does not reduce the synchronization of interneurons in the molecular layer of the cerebellum 
between which electrical synapses have been identified (Mann-Metzer and Yarom 1999). In slices of neocortex, in which LTS cells are activated by ACPD, the synchronous activity of LTS remains after blocking inhibition (Beierlein et al 2000). Reciprocally, blockade of gap-junctions through pharmacological agent (PerezVelazquez et al. 1994; Traub et al. 2001; Friedman and Strowbridge 2003) or genetic manipulations (Deans et al. 2001; Hormuzdi et al. 2001; Blatow et al. 2003) reduces the synchronized activity in cortex or in hippocampus. Other studies suggest that electrical synapses alone are not sufficient for getting synchronous activity but that they are necessary. This has been shown in cortical slice preparations where electrical synapses or inhibitory ones are not able to synchronize neuronal activity (Tamas et al. 2000; Blatow et al. 2003). In contrast with these results, it has been reported that in inspiratory motoneurons synchronous activity depends on inhibition and that it is strongly enhanced in presence of carbenoxolone, a blocker of electrical synapses (Bou-Flores and Berger 2001). Therefore, electrical synapses may also desynchronize neural activity.

These experimental facts raise several issues. How do intrinsic cellular properties affect synchrony of neurons coupled via electrical synapses? How does synchrony depends on the localization of the synapses on the dendrite or the soma (or between axons) (Traub et al. 2001)? What is the significance of the combination of electrical and inhibitory couplings for neuronal dynamics? How does synchrony depends on the firing rates of the neurons? What is nature of the interplay between chemical and electrical synapses in promoting synchrony, in particular, how do inhibition and electrical coupling combine their effects in the dynamics of small and large neural systems?

In this chapter, we address these issues in the framework of the theory of weakly coupled oscillators. The intrinsic dynamics of the neuron can be described in terms of the infinitesimal Phase Response Curve also called infinitesimal Phase Response Function (iPRC). The iPRC can be defined for any dynamical system that displays a stable limit cycle (Kuramoto 1984). It is evaluated by applying a small perturbation at a given phase of the cycle and recording the change of the phase induced by the perturbation. Combining this concept with a weak coupling approximation one can develop very powerful tools to analyze complex dynamical systems with a large number of degrees of freedom. Not only the dimensionality of the problem is reduced, but the approximation gives rise to a theoretical framework in which synchronization properties can be analyzed in a very natural way.

The outline of the chapter is as follows: in the next section, we present the theory of weakly coupled oscillators following an approach developed by Kuramoto (1984). We then apply this theory in the third section to study the dynamics of neuronal networks in which neurons interact via electrical synapses. The results obtained in that framework emphasize how the synchronizing effect of electrical synapses depends on the intrinsic properties of the neurons. The various regimes of the interplay between electrical and inhibitory synapses are discussed in the fourth section. We conclude the chapter by discussing the relevance of the results derived in the framework of weakly coupled oscillators to explain the synchronization behavior observed in more realistic numerical simulations as well as in physiological experiments. 


\section{Phase Models for Networks of Weakly Coupled Neurons}

\subsection{Phase Response Function and iPRC of Intrinsic Oscillators}

Let us consider a nonlinear oscillator whose state is described by the vector $\mathbf{X}$. For instance for the Hodgkin-Huxley (HH) (Hodgkin and Huxley 1952) model these components are $V, m, n, h$, where $V$ is the membrane potential and $m, n, h$ are the gating variables of the potassium and sodium currents. For a multicompartmental neuronal model the dimension of vector $\mathbf{X}$ is larger, because it incorporates variables related to each compartment. This vector evolves according to:

$$
\frac{\mathrm{d} \mathbf{X}}{\mathrm{d} t}=\mathbf{F}(\mathbf{X})
$$

We assume that this system has a limit cycle solution, with period $T(\mathbf{X}(t+T)=$ $\mathbf{X}(t))$ which is asymptotically stable, i.e., that there is a neighborhood $\mathscr{G}$ of the periodic solution such that the dynamical system with any initial condition in $\mathscr{G}$ eventually converges to this solution. For any point on the limit cycle we define a phase function $\phi(\mathbf{X})$. We first choose an arbitrary point $\mathbf{X}_{0}$ and we assign to it a phase variable $\phi\left(\mathbf{X}_{0}\right)=0$. The phase of the other points on the limit cycle is defined via the differential equation:

$$
\frac{\mathrm{d} \phi(\mathbf{X})}{\mathrm{d} t}=1 .
$$

This definition can be extended to points sufficiently close to the limit cycle as follows. Let us consider a point $\mathbf{Y}_{0}$ in $\mathscr{G}$. This point evolves according to (15.1), giving rise to the trajectory $\mathbf{Y}(t)$. By definition of $\mathscr{G}$, this trajectory eventually converges to the limit cycle. Therefore, there is a unique point $\mathbf{X}_{Y}$ on the limit cycle, whose trajectory $\mathbf{X}_{\mathbf{Y}}(t)$ is such that: $\lim _{t \rightarrow \infty}\left|\mathbf{Y}(t)-\mathbf{X}_{Y}(t)\right| \rightarrow 0$. Hence, one can associate a phase variable to $\mathbf{Y}$ as: $\phi(\mathbf{Y}) \equiv \phi\left(\mathbf{X}_{Y}\right)$ and this phase evolves with the same velocity for points on the limit cycle or in its neighborhood, i.e.,

$$
\frac{\mathrm{d} \phi(\mathbf{Y})}{\mathrm{d} t}=1
$$

Let us now consider the perturbed system

$$
\frac{\mathrm{d} \mathbf{X}}{\mathrm{d} t}=\mathbf{F}(\mathbf{X})+\varepsilon \mathbf{p}(\mathbf{X})
$$

If the perturbation is weak enough, $\varepsilon \ll 1$, the trajectory stays in the neighborhood of the unperturbed limit cycle where the phase is still defined. This phase evolves according to:

$$
\frac{\mathrm{d} \phi(\mathbf{X})}{\mathrm{d} t}=\frac{\partial \phi}{\partial \mathbf{X}} \cdot \frac{\mathrm{d} \mathbf{X}}{\mathrm{d} t}=1+\varepsilon \frac{\partial \phi}{\partial \mathbf{X}} \cdot \mathbf{p}(\mathbf{X}) .
$$


This equation controls the evolution of the phase in the basin of attraction of the limit cycle. It is exact but useless for determining the time evolution of the phase, because to evaluate the right hand side of the equation we need to know the point $\mathbf{X}$ at which to evaluate the functions $\frac{\partial \phi}{\partial \mathbf{X}}$ and $\mathbf{p}(\mathbf{X})$. In general, it is not possible to recover that information from the phase $\phi$ alone. However, since $\varepsilon \ll 1$, we can assume that the trajectory remains at a distance of order $\varepsilon$ from the limit cycle. The right hand side can then be estimated on the limit cycle, i.e.,

$$
\frac{\mathrm{d} \phi}{\mathrm{d} t}=1+\varepsilon \mathbf{Z}(\phi) \cdot \mathbf{p}(\phi)+O\left(\varepsilon^{2}\right)
$$

with:

$$
\mathbf{Z}(\phi)=\frac{\partial \phi(\mathbf{X})}{\partial \mathbf{X}}
$$

and $\mathbf{p}(\phi)=\mathbf{p}(\mathbf{X})$ for a point $\mathbf{X}$ whose phase is $\phi$.

The vector $\mathbf{Z}(\phi)$ is the infinitesimal phase response function of the oscillator. It characterizes the change of phase induced by a weak perturbation. Typically, the synaptic input from other neurons $\mathbf{p}$ affects only the voltage component of the state space. Therefore, we will usually consider only the voltage component of the phase response function, which defines an iPRC Curve. There are only few examples of nonlinear oscillators for which the limit cycle and the iPRC can be computed analytically (see some examples below). In general, numerical methods have to be used. One approach is to integrate numerically the dynamics to compute the limit cycle over one period and subsequently the derivative of the phase is evaluated by estimating the dephasing induced at large time by small initial perturbations at different points covering the limit cycles. This approach which is directly related to the definition of the iPRC is intuitive but not rigorous. In particular, it is necessary to check that the amplitude of the perturbation which is used is sufficiently small. This method was applied for instance in Hansel et al. (1993). Another method, more rigorous mathematically but less intuitive, is introduced in Ermentrout and Kopell (1992); Kopell (1998). It is implemented in the software XPP (Ermentrout 2003).

Knowing this, iPRC allows one to compute how the phase of the oscillator evolves under small perturbations. For any time-dependent function $x$, we define the time average over one period

$$
<x>(t)=\frac{1}{T} \int_{t-T}^{t} x\left(t^{\prime}\right) \mathrm{d} t^{\prime} .
$$

Let us note that for a weakly perturbed oscillator, the time average differs from the function itself by an amount of order $\varepsilon$ (Kuramoto 1984). The main reason for introducing the time average is that, as we will see below, in terms of this new variable the dynamics of a system of coupled oscillators depends only on the phase differences. Integrating (15.6) between $[t-T, t]$, we obtain the evolution of the time averaged phase:

$$
\frac{\mathrm{d}<\phi>(t)}{\mathrm{d} t}=1+\frac{\varepsilon}{T} \int_{t-T}^{t} \mathbf{Z}\left(\phi\left(t^{\prime}\right)\right) \cdot \mathbf{p}\left(\phi\left(t^{\prime}\right)\right) \mathrm{d} t^{\prime}+O\left(\varepsilon^{2}\right) .
$$


As $\phi$ and $\langle\phi>$ differ at most by an amount of order $\varepsilon$ and we can replace $\phi$ by $<\phi>$ in the right hand side:

$$
\frac{\mathrm{d}<\phi>(t)}{\mathrm{d} t}=1+\frac{\varepsilon}{T} \int_{t-T}^{t} \mathbf{Z}\left(<\phi\left(t^{\prime}\right)>\right) \cdot \mathbf{p}\left(<\phi\left(t^{\prime}\right)>\right) \mathrm{d} t^{\prime}+O\left(\varepsilon^{2}\right) .
$$

If we change units such that the phase $\phi$ goes from 0 to $2 \pi$, we have

$$
\frac{\mathrm{d} \phi}{\mathrm{d} t}=\omega+\frac{\varepsilon}{2 \pi} \int_{0}^{2 \pi} \mathbf{Z}(\phi) \cdot \mathbf{p}(\phi) \mathrm{d} \phi+O\left(\varepsilon^{2}\right),
$$

where $\omega=2 \pi / T=2 \pi f$ and $\mathbf{Z}(\phi)$ and $\mathbf{p}(\phi)$ are $2 \pi$-periodic functions of $\phi$. We have also dropped the brackets for simplicity. In the following we will be always writing the dynamics in terms of time averaged quantities.

\subsection{Examples of iPRC of Neurons}

The iPRC, which characterizes how neurons respond to small perturbations that affect its voltage component, is a key concept to understand the relationship between intrinsic properties of neurons and their collective dynamics (Hansel et al. 1993; van Vreeswijk et al. 1994; Kopell and Ermentrout 2002). The iPRC depends on the excitability properties of the neurons and therefore on the intrinsic currents involved in their dynamics.

\subsection{1 iPRC of Conductance-Based Neurons}

Figure 15.1 plots the iPRCs for the HH model and the Wang-Buzsáki (WB) model (two models which are frequently used in neural modeling). Both models involve a potassium delayed rectifier current and a sodium current. However, the parameters of these currents are not the same in the two models leading to excitability properties which differ qualitatively. In particular, the onset of firing is a subcritical Hopf bifurcation in the $\mathrm{HH}$ model whereas in the WB model it is a saddle-node bifurcation. Accordingly, the $\mathrm{HH}$ neuron has a minimal firing frequency in contrast to the WB neuron that is capable of firing at arbitrary low frequency in response of an external steady state current sufficiently close to the rheobase. The two models display also qualitative differences in their iPRCs. The iPRC of the WB neurons is positive everywhere on the limit cycle except in a very small region which coincides with the action potential. This means that the WB neuron responds to small depolarizations by advancing its phase. This is in contrast to what is found in the $\mathrm{HH}$ neuron where on a substantial range of the limit cycle the iPRC is negative. Another difference is that in a region of the limit cycle the iPRC of the HH neuron is close to zero indicating that the neuron is insensitive to perturbations in that region. 
Fig. 15.1 iPRCs (top panels) and voltage traces (middle panels) during one period of oscillation for Wang-Buzsáki neuron (left) and

Hodgkin-Huxley neuron (right). Bottom panels show iPRCs for the normal form of Type I neuron (left) and Type II neuron (right). $\phi=0$ corresponds to the phase at which the membrane potential crosses $V=0$ upwards
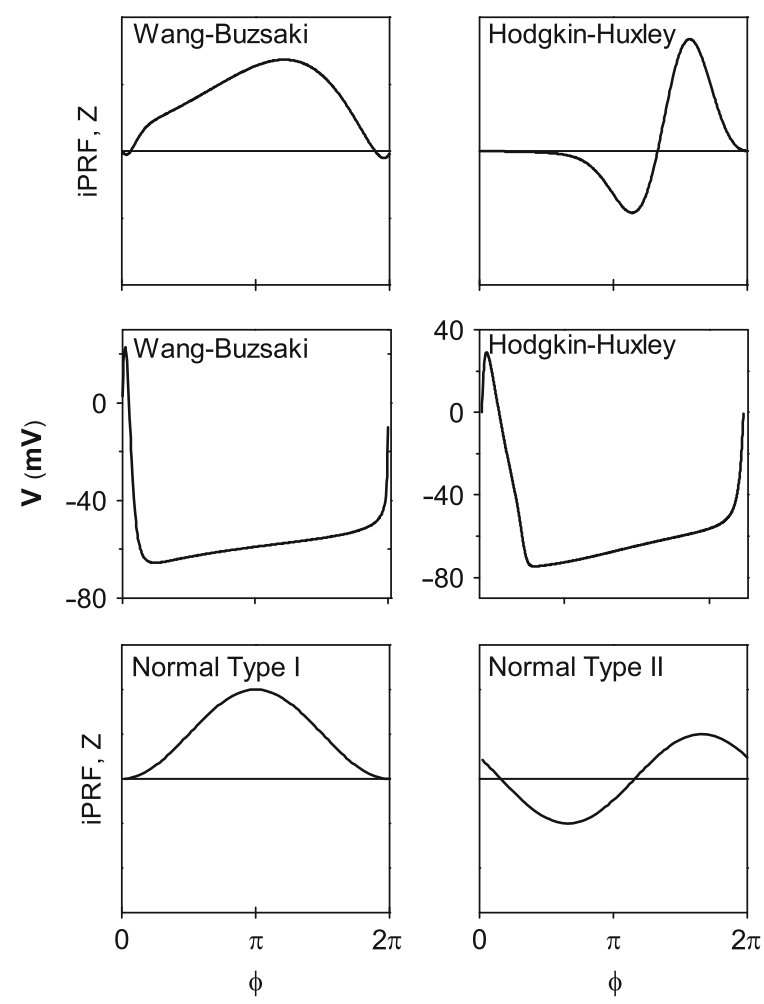

This last feature is related to the fact that the action potential is substantially broader for the HH model than for the WB model. In Fig. 15.4, we display other examples of iPRC obtained in conductance-based neurons showing how intrinsic currents shape the response to small perturbation.

The role of the intrinsic currents in shaping the iPRC can be studied analytically when the external current induces periodic spiking near the firing onset. This is because near this onset the neural dynamics can be assimilated to their normal forms, which depend only on the bifurcation structure. In Brown et al. (2004), the iPRCs have been evaluated for the four codimension one bifurcations. The more typical cases found in neural modeling are the saddle node, or Type I, and the subcritical Hopf, or Type II, bifurcations. One can show that for Type I models, the iPRC is given by:

$$
Z(\phi) \propto 1-\cos (\phi),
$$

while for Type II models it is

$$
Z(\phi) \propto \sin \left(\phi-\phi_{H}\right),
$$


where that phase variable $\phi$ goes from 0 to $2 \pi$ and the constant $\phi_{H}$ can be derived from the original equations of the model. It is important to remark that in the second case the iPRC of the full model will approach to the result of (15.13) only if the radius of the oscillation near the bifurcation is small (Brown et al. 2004). This is not the case in the $\mathrm{HH}$ model, therefore the relation between the iPRC of the full model and the iPRC of the normal form is only qualitative. In contrast, for the Type I model, the two iPRCs agree more at lower firing rates. However, the iPRCs of the $\mathrm{HH}$ model and the normal form of the Type II models share an important feature: the iPRC has a significant negative region before the positive region (see Fig. 15.1).

\subsection{2 iPRC of Integrate-and-Fire Neurons}

In integrate-and-fire model of neurons, the subthreshold membrane potential follows the dynamics equation:

$$
\tau_{0} \frac{\mathrm{d} v}{\mathrm{~d} t}=f(v)+I_{\mathrm{ext}},
$$

where $\tau_{0}$ is the time constant of the dynamics. These dynamics are supplemented by the condition that whenever the membrane potential reaches a threshold $V_{\mathrm{T}}$, an action potential is fired and $v$ is reset to a potential $V_{\mathrm{r}}$. The membrane potential of the neurons can be written as the sum of its subthreshold component $v(t)$ and a $\delta$ function which represents the suprathreshold component (i.e., the action potential):

$$
V(t)=v(t)+\theta \sum_{\text {spikes }} \delta\left(t-t_{\text {spike }}\right),
$$

where $\theta$ measures the integral over time of the supra-threshold part and $t_{\text {spike }}$ satisfies $v\left(t_{\text {spike }}\right)=V_{\mathrm{T}}$.

For one-dimensional models, the trajectory unequivocally determines the iPRC. It can be written as (Kuramoto 1984; Hansel et al. 1995):

$$
Z(\phi)=\left(\frac{\mathrm{d} v(\phi)}{\mathrm{d} \phi}\right)^{-1}
$$

In the standard leaky integrate-and-fire (LIF) model:

$$
f(v)=-\left(v-V_{\mathrm{L}}\right)
$$

where $V_{\mathrm{L}}$ is the reversal potential of the leak current. The iPRC can be easily calculated. One finds:

$$
Z(\phi)=\frac{2 \pi \tau_{0}}{T\left(I_{\mathrm{ext}}+V_{\mathrm{L}}-V_{\mathrm{r}}\right)} \exp \left(\frac{\phi T}{2 \pi \tau_{0}}\right),
$$


where the firing period is defined as $2 \pi$. The iPRC of LIF neurons is a monotonically increasing exponential function, which does not capture nonmonotonic behavior of iPRC observed in type-I conductance-based neuronal model.

Nonlinear integrate-and-fire models may provide a more accurate description of the membrane subthreshold dynamics that take into account some basic nonlinearities induced by voltage-dependent currents. A special case is the quadratic integrate-and-fire (QIF) model whose membrane time course and iPRC can be computed analytically. Its membrane voltage evolves in time:

$$
\tau_{0} \frac{\mathrm{d} v}{\mathrm{~d} t}=\left(v-V_{\mathrm{s}}\right)^{2}+I_{\mathrm{ext}},
$$

where $V_{\mathrm{s}}$ is the value of potential membrane at the equilibrium point $\left(I_{\text {ext }}=0\right)$.

Integrating (15.19), one finds that the general solution for the subthreshold potential is for $I_{\text {ext }}>0$ :

$$
v(t)=V_{\mathrm{s}}+\sqrt{I_{\mathrm{ext}}} \tan \left(\frac{t \sqrt{I_{\mathrm{ext}}}}{\tau_{0}}+\alpha\right),
$$

where $\alpha$ is a constant of integration. The condition that at $t=0$ the membrane potential of the neuron is at its reset value, $V_{\mathrm{r}}$, determines $\alpha$ :

$$
\alpha=\tan ^{-1}\left(\frac{V_{\mathrm{r}}-V_{\mathrm{s}}}{\sqrt{I_{\mathrm{ext}}}}\right) .
$$

The function $v(t)$ increases monotonically to infinity. Therefore, starting from the resetting potential $V_{\mathrm{r}}, v(t)$ reaches the threshold after some time $T$. The condition $v(T)=V_{\mathrm{T}}$ determines the firing period. One finds:

$$
T=\tau_{0} \frac{\tan ^{-1}\left(\frac{V_{\mathrm{T}}-V_{\mathrm{s}}}{\sqrt{I_{\mathrm{ext}}}}\right)-\tan ^{-1}\left(\frac{V_{\mathrm{r}}-V_{\mathrm{s}}}{\sqrt{I_{\mathrm{ext}}}}\right)}{\sqrt{I_{\mathrm{ext}}}} .
$$

The f-I curve of the QIF neuron is given by $f=1 / T\left(I_{\text {ext }}\right)$. Let us note that a continuous f-I curve for small $I-I_{\text {ext }}$ requires that the parameters $V_{\mathrm{T}}-V_{\mathrm{s}}$ and $V_{\mathrm{r}}-V_{\mathrm{s}}$ have opposite sign. As $V_{\mathrm{T}}$ is always larger than $V_{\mathrm{s}}$ we must take $V_{\mathrm{T}}>V_{\mathrm{s}}$ and $V_{\mathrm{r}}<V_{\mathrm{s}}$.

In the limit $V_{\mathrm{T}} \rightarrow \infty, V_{\mathrm{r}} \rightarrow-\infty$ or in the limit of small firing rate, the QIF model reduces to the theta model (Ermentrout and Kopell 1986; Ermentrout 1996). This model describes the normal form of the oscillatory dynamics appearing after a saddle-node bifurcation (Brown et al. 2004; Ermentrout 1996) that characterizes Type I neurons (see Hansel and Mato 2003 for the details of the derivation).

The iPRC of QIF neurons can be computed analytically by combining (15.16) and (15.19) with $\phi=2 \pi t / T$ :

$$
Z(\phi)=\frac{\tau_{0}}{\left[v\left(\frac{\phi T}{2 \pi}\right)-V_{\mathrm{s}}\right]^{2}+I_{\mathrm{ext}}} .
$$


a
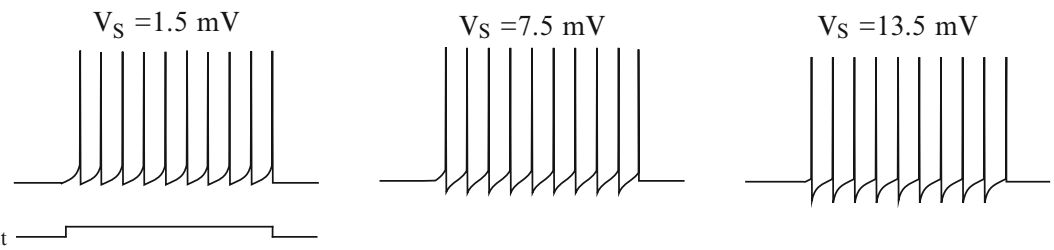

b
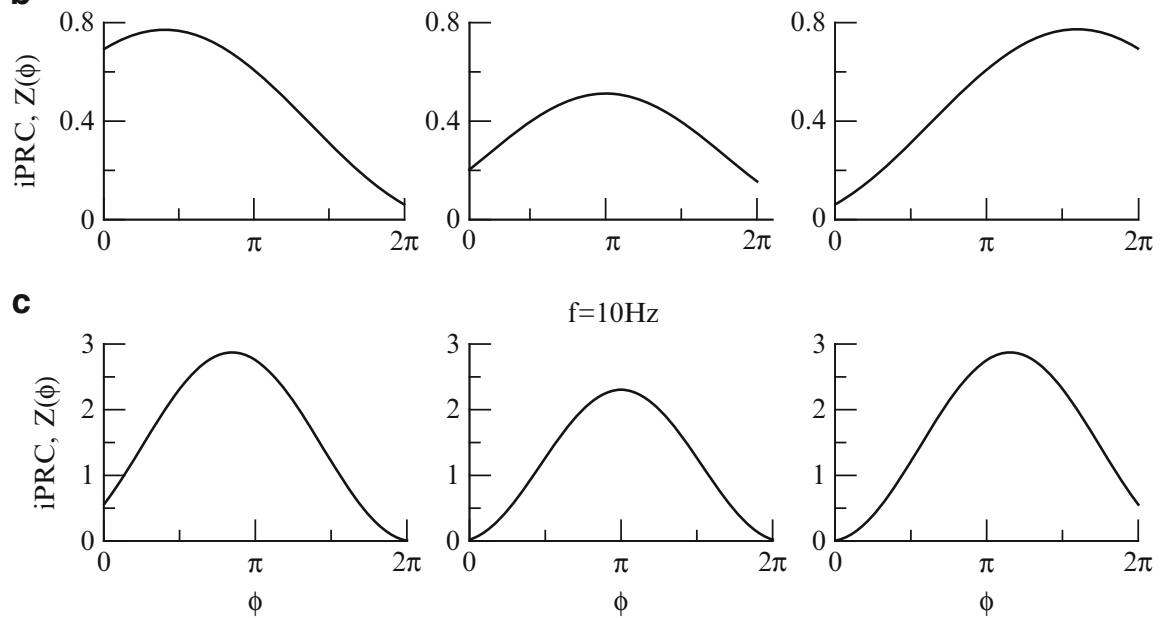

Fig. 15.2 Voltage traces and iPRCs of the QIF neuron. The voltage traces and the iPRCs were computed using (15.15) and (15.24), respectively (with $\left.\tau_{0}=10 \mathrm{~ms}\right)$. In (a) and (b), the external current is such that the firing rate is $50 \mathrm{~Hz}$. (a), The voltage trace of the neuron in response to a constant injected current for three values of the ratio $V_{\mathrm{s}}$. The reset depth is the same in all three cases: $V_{\mathrm{T}}-V_{\mathrm{r}}=15 \mathrm{mV}$. Note the changes in the concavity of the voltage trace between the spikes when $V_{\mathrm{s}}$. (b), The iPRC, $Z$, for the same cases as in (a). (c), Same as in (b) but for a firing rate of $10 \mathrm{~Hz}$. The phase response changes less than in (b) when $V_{\mathrm{s}}$ varies. Similar cases are also shown in Chap. 14

Replacing the membrane potential from (15.20), we finally find

$$
Z(\phi)=\frac{\tau_{0}}{I_{\mathrm{ext}}} \cos ^{2}\left(\frac{\phi T \sqrt{I_{\mathrm{ext}}}}{2 \pi \tau_{0}}\right) .
$$

In the following sections, we always take $V_{\mathrm{r}}=0$ and $V_{\mathrm{T}}=15 \mathrm{mV}$. The parameter $V_{\mathrm{s}}$ controls the shape of the subthreshold trajectory and the iPRC, as shown in Fig. 15.2. Figure 15.2a plots the membrane potential of a QIF neuron firing at $50 \mathrm{~Hz}$ for different values of $V_{\mathrm{s}}$. For $V_{\mathrm{s}}=\left(V_{\mathrm{T}}+V_{\mathrm{r}}\right) / 2$, the concavity changes from upward to downward at the middle of the period. Taking more (resp. less) positive values of $V_{\mathrm{s}}$ this inflexion point shifts to the left (resp. right).

Figure $15.2 \mathrm{~b}$ plots the iPRC for the same values of $V_{\mathrm{s}}$ and $I_{\mathrm{ext}}$. As it is obvious from (15.24), $Z(\phi)>0$ for all $\phi$ and has only one maximum. The location of the 
maximum of $Z$ depends on $V_{\mathrm{s}}$ and $I_{\text {ext }}$. For $V_{\mathrm{s}}<\left(V_{\mathrm{T}}+V_{\mathrm{r}}\right) / 2$, it is located in the first half of the firing period, whereas for $V_{\mathrm{s}}>\left(V_{\mathrm{T}}+V_{\mathrm{r}}\right) / 2$ it is located in the second half. The larger the firing rate, the stronger the dependence of $Z$ on $V_{\mathrm{s}}$. For $V_{\mathrm{s}}=\left(V_{\mathrm{T}}+V_{\mathrm{r}}\right) / 2, Z(\phi)$ is symmetric and its maximum is always at $\phi=\pi$.

Expressions for the iPRC of LIF and QIF neurons are also given in Chap. 14, although in that case there is no rescaling of the period from $T$ to $2 \pi$.

\subsection{Phase Dynamics of a Networks of Weakly Coupled Neurons}

We consider now a network of $N$ identical neurons interacting in a pairwise manner. The dynamics of neuron $l$ has the form:

$$
\frac{\mathrm{d} \mathbf{X}_{l}}{\mathrm{~d} t}=\mathbf{F}\left(\mathbf{X}_{l}\right)+g \sum_{j=1}^{N} J_{l j} \mathbf{G}\left(\mathbf{X}_{l}, \mathbf{X}_{j}\right)+\mathbf{s}_{l}(t)
$$

where $J_{l j}$ is the connectivity matrix: $J_{l j}=1$ if the presynaptic neuron $j$ interacts with the neuron $l$ and $J_{l j}=0$, otherwise. $\mathbf{G}$ is the synaptic interaction which depends on the state of presynaptic and postsynaptic neurons. The strength of the synapses is denoted by $g$. We also assume that the neurons receive a Gaussian white noise input that affects only the voltage component $\left(\left(\mathbf{s}_{l}\right)_{j}=0\right)$ for $j \neq 1$ with: $<\left(\mathbf{s}_{l}\right)_{1}>=0,<\left(\mathbf{s}_{l}(t)\right)_{1}\left(\mathbf{s}_{m}\left(t^{\prime}\right)\right)_{1}>=2 \sigma^{2} \delta_{l, m} \delta\left(t-t^{\prime}\right)$, where $\delta_{l, m}=1$ for $l=m$ and $\delta_{l, m}=0$ for $l \neq m$. This means that the noise is uncorrelated in time and across neurons. This equation has the form of (15.4). When the coupling strength is sufficiently weak ( $g \ll 1$ and $\sigma^{2} \approx g$ ), one can use the weak coupling theory developed above to reduce the dynamics of (15.25) to the dynamics of a network of phase oscillators. One finds:

$$
\frac{\mathrm{d} \phi_{l}}{\mathrm{~d} t}=\omega+\sum_{j=1}^{N} J_{l j} \Gamma\left(\phi_{l}-\phi_{j}\right)+\eta_{l}(t),
$$

where

$$
\Gamma(\phi)=\frac{g}{2 \pi} \int_{0}^{2 \pi} \mathbf{Z}\left(\phi^{\prime}+\phi\right) \cdot \mathbf{p}\left(\phi^{\prime}+\phi, \phi^{\prime}\right) \mathrm{d} \phi^{\prime},
$$

and $\eta_{l}(t)$ is the effective noise in the phase dynamics.

The function $\Gamma(\phi)$ is the phase interaction function. It is a convolution between the phase response function $\mathbf{Z}(\phi)$ and the perturbation induced by the interactions, $\mathbf{p}\left(\phi_{1}, \phi_{2}\right)=\mathbf{G}\left(\mathbf{X}\left(\phi_{1}\right), \mathbf{X}\left(\phi_{2}\right)\right)$ evaluated on the limit cycle trajectory of presynaptic and postsynaptic neurons. It is a periodic function with period $2 \pi$ because both $\mathbf{Z}(\phi)$ and $\mathbf{p}\left(\phi, \phi^{\prime}\right)$ are periodic with that period. 
Generalizing the approach developed by Kuramoto (1991) in the context of the LIF model, one can show that $\eta(t)$ is white Gaussian noise with zero mean and variance $D$ :

$$
D=\frac{\sigma^{2}}{2 \pi} \int_{0}^{2 \pi}\left[(\mathbf{Z}(\phi))_{1}\right]^{2} \mathrm{~d} \phi .
$$

Therefore, the effective noise in the phase dynamics of a neuron is proportional to the average of the square of its iPRC over one period.

In the following, we consider two types of neuronal systems, (1) a pair of coupled neurons and (2) a network of $N$ all-to-all coupled neurons. Let us note that the first case is also covered in Chap. 14, but not the second one. They also do not analyze the effect of the noise.

\subsubsection{Cross-Correlation of a Pair of Neurons in Presence of Weak Noise}

The cross-correlation (CC) of the firing activity of pairs of interacting neurons measures the probability that the two neurons fire with some given time delay. This is a standard tool for evaluating the synchronization between pairs of neurons. Here, we derive an analytical expression for the $\mathrm{CC}$ of a pair of spiking neurons under the assumptions of weak interactions and weak noise. It provides valuable information about the way how noise and intrinsic and synaptic properties affect the shape of the $\mathrm{CC}$. In the presence of weak noise, the dynamics of two coupled identical oscillators is given by

$$
\begin{aligned}
\frac{\mathrm{d} \phi_{1}}{\mathrm{~d} t} & =\omega+\Gamma\left(\phi_{1}-\phi_{2}\right)+\eta_{1}(t), \\
\frac{\mathrm{d} \phi_{2}}{\mathrm{~d} t} & =\omega+\Gamma\left(\phi_{2}-\phi_{1}\right)+\eta_{2}(t) .
\end{aligned}
$$

The time elapsed at time $t$ since the last spike fired by neuron $i$ is simply the firing period, $T$, multiplied by the phase, $\phi(t)$, computed modulo $2 \pi\left(\bmod \left(\phi_{i}(t), 2 \pi\right)\right)$ and divided by $2 \pi$. Similarly, the time elapsed between the last spikes fired by neurons $i$ and $j$ is: $\tau=T \bmod \left(\phi_{i}(t)-\phi_{j}(t), 2 \pi\right) /(2 \pi)$. In the weak coupling limit, the CC can be evaluated in terms of the probability distribution function of phase shifts. We define the joint probability $P_{1,2}\left(t_{1}, t_{2}\right)$ as the probability that neuron 1 fires between $t_{1}$ and $t_{1}+\delta$ and neuron 2 fires between $t_{2}$ and $t_{2}+\delta$. The spike train of neuron 1 is $S_{1}(t)$, i.e., $S_{1}(t)=1$ if there is a spike in the bin $[t, t+\delta]$ and $S_{1}(t)=0$ if there is no spike. The time average (over the whole spike train) is $<S_{1}(t)>\equiv P_{1}(t) . P_{1}(t)$ does not depend on time and it is equal to $f=\frac{\omega}{2 \pi}$. Using Bayes theorem, we find

$$
<S_{1}\left(t_{1}\right) S_{2}\left(t_{2}\right)>=P_{1,2}\left(t_{1}, t_{2}\right)=P_{2 \mid 1}\left(t_{2} \mid t_{1}\right) P_{1}\left(t_{1}\right)
$$


where $P_{2 \mid 1}$ is the conditional probability that neuron 2 fires between $t_{2}$ and $t_{2}+\delta$ given that neuron 1 fired between $t_{1}$ and $t_{1}+\delta$. Assuming stationarity, this function depends only on the difference $\tau=t_{2}-t_{1}: P_{2 \mid 1}\left(t_{2} \mid t_{1}\right)=\tilde{P}_{2 \mid 1}(\tau)$, and hence

$$
\frac{<S_{1}\left(t_{1}\right) S_{2}\left(t_{1}+\tau\right)>}{<S_{1}(t)><S_{2}(t)>}=\frac{\tilde{P}_{2 \mid 1}(\tau)}{\omega} .
$$

Switching from a function of time to a function of phase, we obtain

$$
C(\tau) \equiv \frac{<S_{1}\left(t_{1}\right) S_{2}\left(t_{1}+\tau\right)>}{<S_{1}(t)><S_{2}(t)>}=2 \pi P_{0}(\phi),
$$

where $P_{0}$ is the probability distribution of the phase shift between the two neurons.

Using (15.29) and (15.30) one finds that $\Delta=\phi_{1}-\phi_{2}$ satisfies the Langevin equation:

$$
\frac{\mathrm{d} \Delta}{\mathrm{d} t}=\Gamma_{-}(\Delta)+\eta(t),
$$

where we have defined $\eta(t) \equiv \eta_{1}(t)-\eta_{2}(t)$ and $\Gamma_{-}(\Delta) \equiv(\Gamma(\Delta)-\Gamma(-\Delta))$.

The noise $\eta(t)$ is the difference of two Gaussian white noises, with zero average and variance $D$. Therefore, it is a Gaussian white noise with zero average and variance $2 D$. Hence, the phase-shift distribution, $P(\Delta, t)$, satisfies a Fokker-Planck equation (van Kampen 1981):

$$
\frac{\partial P(\Delta, t)}{\partial t}=D \frac{\partial^{2}}{\partial \Delta^{2}} P(\Delta, t)-\frac{\partial}{\partial \Delta}\left[\Gamma_{-}(\Delta) P(\Delta, t)\right] .
$$

The stationary distribution of the phase shifts, $P_{0}$, is the solution of this equation which satisfies the additional constraint

$$
\frac{\partial P_{0}(\Delta, t)}{\partial t}=0
$$

that is:

$$
P_{0}(\Delta)=\mathrm{e}^{G(\Delta)}\left[A \int_{0}^{\Delta} \mathrm{e}^{-G\left(\Delta^{\prime}\right)} \mathrm{d} \Delta^{\prime}+B\right]
$$

where:

$$
G(\Delta)=\int_{0}^{\Delta} \frac{\Gamma_{-}\left(\Delta^{\prime}\right)}{D} \mathrm{~d} \Delta^{\prime}
$$

and $A$ and $B$ are two integration constants which are determined by the periodicity of $P_{0}$, i.e., $P_{0}(0)=P_{0}(2 \pi)$, and its normalization, i.e., $\int_{0}^{2 \pi} P_{0}(\Delta) \mathrm{d} \Delta=1$. One finds:

$$
P_{0}(\Delta)=\frac{\mathrm{e}^{G(\Delta)}}{\int_{0}^{2 \pi} \mathrm{e}^{G\left(\Delta^{\prime}\right)} \mathrm{d} \Delta^{\prime}} .
$$

One can show that $P_{0}$ has an extremum for phase shifts $\Delta$ that are solutions of:

$$
\Gamma_{-}(\Delta)=0 .
$$


These solutions for which

$$
\frac{\mathrm{d} \Gamma_{-}}{\mathrm{d} \phi}(\Delta)=\Gamma^{\prime}(\Delta)<0(\text { resp. }>0)
$$

are maxima (resp. minima) of $P_{0}$. Equations (15.40) and (15.41) can be easily interpreted. Indeed, in the absence of noise, the two neurons are phase locked at large time and the possible phase shifts are the stable fixed point of (15.34) with $\eta=0$. It is straightforward to see that these phase shifts are the solutions of (15.40) and that (15.41) is the condition for their stability. In presence of noise, the phase shifts between the two neurons have an increase (resp. decrease) in probability density to be around the stable (resp. unstable) fixed points of the noiseless dynamics. In the limit $D \rightarrow 0, P_{0}(\Delta)$ is nonnegligible only for values of $\Delta$ in the vicinity of the maxima of $G(\Delta)$. In general, $G(\Delta)$ is continuously differentiable at least up to the second order. Expanding $G$ around its maxima, one finds that $P_{0}$ is a sum of Gaussians centered around these points, with a width proportional to $1 / \sqrt{-\Gamma^{\prime}(\Delta)}$. Therefore, the greater the stability is of a fixed point in absence of noise, the sharper the probability distribution is when noise is present.

\subsubsection{Stability of the Asynchronous State in Large Neuronal Network in Presence of Noise}

We now consider the phase dynamics (15.26), with $N \gg 1$ and all-to-all coupling. The stability analysis of the asynchronous state can be investigated for a network of phase oscillators in presence of white noise. The dynamics is given by:

$$
\frac{\mathrm{d} \phi_{l}}{\mathrm{~d} t}=\omega+\sum_{j=1}^{N} \Gamma\left(\phi_{l}-\phi_{j}\right)+\eta_{l}(t),
$$

where $\eta_{i}$ are independently distributed white noise with Gaussian distribution (zero mean and variance $D$ ). Note that in order to have a well-defined limit, $N \rightarrow \infty$, the interaction strength has to scale as $1 / N$.

Let us define $n(\phi, t)$ as the fraction of oscillators that have a given phase at a given time. This quantity evolves according to a Fokker-Planck equation (van Kampen 1981):

$$
\frac{\partial n(\phi, t)}{\partial t}+\frac{\partial(v(\phi, t) n(\phi, t))}{\partial \phi}=D \frac{\partial^{2} n(\phi, t)}{\partial^{2} \phi}
$$

with

$$
v(\phi, t)=\omega+\int_{0}^{2 \pi} \Gamma\left(\phi-\phi^{\prime}\right) n\left(\phi^{\prime}, t\right) \mathrm{d} \phi^{\prime} .
$$


$v(\phi, t)$ represents the velocity of the oscillators that have a phase $\phi$ at time $t$. This equation always has a solution $n(\phi, t)=N /(2 \pi)$. This solution corresponds to the asynchronous state of the network in which the probability of firing of the neurons in the network is constant in time. In this state, the temporal fluctuation of any population average observable vanishes in the large $N$ limit.

To analyze the stability of this state, we expand $n(\phi, t)$ in the vicinity of the asynchronous state solution:

$$
n(\phi, t)=\frac{N}{2 \pi}+\varepsilon(\phi, t)
$$

Inserting this expression into the Fokker-Planck equation we obtain (up to order $\left.\varepsilon^{2}\right)$ :

$$
\frac{\partial \varepsilon(\phi, t)}{\partial t}+\frac{\partial\left(\left(\omega+N \Gamma_{0}\right) \varepsilon(\phi, t)+u\right)}{\partial \phi}=D \frac{\partial^{2} \varepsilon(\phi, t)}{\partial^{2} \phi}
$$

with $u=\frac{N}{2 \pi} \int_{0}^{2 \pi} \Gamma\left(\phi-\phi^{\prime}\right) \varepsilon\left(\phi^{\prime}, t\right) \mathrm{d} \phi^{\prime}$ and $\Gamma_{0}=\frac{1}{2 \pi} \int_{0}^{2 \pi} \Gamma(\phi) \mathrm{d} \phi$. This is a linear partial differential equation that can be transformed in a set of uncoupled linear ordinary differential equations by introducing a Fourier representation. Defining

$$
\begin{aligned}
\varepsilon_{l}(t) & =\frac{1}{2 \pi} \int_{0}^{2 \pi} \varepsilon(\phi, t) \exp (i l \phi) \mathrm{d} \phi \\
\Gamma_{l} & =\frac{1}{2 \pi} \int_{0}^{2 \pi} \Gamma(\phi) \exp (i l \phi) \mathrm{d} \phi
\end{aligned}
$$

we find

$$
\frac{\mathrm{d} \varepsilon_{l}(t)}{\mathrm{d} t}-i l\left(\left(\omega+N \Gamma_{0}\right) \varepsilon_{l}(t)+N \varepsilon_{l}(t) \Gamma_{l}\right)=-l^{2} D \varepsilon_{l}(t)
$$

Looking for solution of the form $\varepsilon_{l}(t) \propto \exp \left(\lambda_{l} t\right)$, one finds that the eigenvalues $\lambda_{l}$ are given by:

$$
\lambda_{l}=i l\left(\left(\omega+N \Gamma_{0}\right)+N \Gamma_{l}\right)-l^{2} D .
$$

The asynchronous state is stable if any small perturbation of it eventually decays to zero. The condition for this stability is therefore:

$$
\operatorname{Re}\left(\lambda_{l}\right)=-l N \operatorname{Im}\left(\Gamma_{l}\right)-l^{2} D \leq 0
$$

for all $l$. 


\section{Dynamics of Neurons Coupled with Electrical Synapses}

An electrical synapse induces a synaptic current proportional to the difference of the membrane potentials of the two coupled neurons. Therefore, one may expect that electrical interactions always tend to equalize the membrane potentials favoring synchrony. However, the actual effect of electrical synapses is more subtle. For instance, it has been found that for pairs of identical neurons coupled with electrical interactions both in-phase and antiphase states can be stable (Han et al. 1995; Chow and Kopell 2000; Lewis and Rinzel 2003). One reason for this complexity is that the current generated by the interaction is filtered in the postsynaptic neuron. Depending on this effect it is possible that the positive current induced by a sharp spike could have less effect than the negative current induced by the after-spike hyperpolarization. Therefore, the temporal course of the membrane potential and the location of the synapse (that affects the filtering properties) can have a strong influence on the net effect of the interaction. In the following, we apply the phase reduction approach to clarify how electrical synapses affect synchrony of neurons.

\subsection{Synchrony of a Pair of Neurons Electrically Coupled}

As shown in Sect. 2, the phase interaction function can be evaluated as a convolution between the iPRC and the synaptic current:

$$
\Gamma\left(\phi_{l}-\phi_{j}\right)=\frac{1}{2 \pi} \int_{0}^{2 \pi} Z\left(u+\phi_{l}\right) I_{\mathrm{syn}}\left(u+\phi_{l}, u+\phi_{j}\right) \mathrm{d} u,
$$

where $Z$ is the iPRC and $I_{\text {syn }}\left(\phi_{l}, \phi_{j}\right)$ is the synaptic current coming from the presynaptic neuron $j$ to the postsynaptic neuron $l$. For electrical synapses, this current is:

$$
I_{\text {syn }}\left(\phi_{l}, \phi_{j}\right)=-g\left(V\left(\phi_{l}\right)-V\left(\phi_{j}\right)\right) .
$$

According to (15.52), the effective phase interaction between two neurons coupled electrically is therefore:

$$
\Gamma(\phi)=\frac{g}{2 \pi} \int_{0}^{2 \pi} Z(u)(V(u-\phi)-V(u)) \mathrm{d} u .
$$

Using this function, we can evaluate the quantities needed to determine the stability of in-phase and antiphase locking of a pair of neurons as well as the asynchronous state of a large network.

The stability of state in which the phase shift between two neurons is $\Delta$ depends on the derivative of the function $\Gamma^{\prime}(\Delta)$. This quantity depends on the derivative of the membrane potential $V(t)$. From (15.15), the membrane potential can be written as the sum of three contributions: 
1. A subthreshold part, during which the membrane potential increases with time from a value $V_{\mathrm{r}}$ to a threshold value $V_{\mathrm{T}}$.

2. A resetting, where potential is instantaneously brought back from $V_{\mathrm{T}}$ to $V_{\mathrm{r}}$.

3. A spike, modeled by a $\delta$-function of amplitude $\theta$.

Differentiating (15.54) with respect to $\phi$ one finds

$$
\Gamma^{\prime}(\phi)=-\frac{g}{2 \pi} \int_{0}^{2 \pi} Z(u) V^{\prime}(u-\phi) \mathrm{d} u .
$$

After doing a change of variables from time to phase and expanding the membrane potential $V$ in its components (spike, reset, and subthreshold component), we have

$$
V^{\prime}(u)=2 \pi f \theta \delta^{\prime}(u)+\left(V_{\mathrm{r}}-V_{\mathrm{T}}\right) \delta(u)+v^{\prime}(u),
$$

where $f$ is the frequency. This allows us to rewrite $\Gamma^{\prime}(\Delta)$ :

$$
\begin{gathered}
\Gamma^{\prime}(\Delta)=-\frac{g}{2 \pi}\left(2 \pi f \theta \int_{0}^{2 \pi} Z(u) \delta^{\prime}(u-\Delta) \mathrm{d} u+\left(V_{\mathrm{r}}-V_{\mathrm{T}}\right) \int_{0}^{2 \pi} Z(u) \delta(u-\Delta) \mathrm{d} u\right. \\
\left.+\int_{0}^{2 \pi} Z(u) v^{\prime}(u-\Delta) \mathrm{d} u\right) .
\end{gathered}
$$

Integrating by parts the first term and using the properties of the $\delta$-function, we finally obtain:

$$
\Gamma^{\prime}(\Delta)=g\left(S_{\mathrm{sp}}+S_{\mathrm{r}}+S_{\mathrm{sub}}\right)
$$

where

$$
\begin{aligned}
S_{\mathrm{sp}} & =f \theta \frac{d Z}{d \phi}(\Delta), \\
S_{\mathrm{r}} & =\frac{1}{2 \pi}\left(V_{\mathrm{T}}-V_{\mathrm{r}}\right) Z(\Delta), \\
S_{\mathrm{sub}} & =-\frac{1}{2 \pi} \int_{0}^{2 \pi} Z(\phi) \frac{\mathrm{d} v}{\mathrm{~d} \phi}(\phi-\Delta) \mathrm{d} \phi .
\end{aligned}
$$

The first term, $S_{\mathrm{sp}}$, is the contribution of the presynaptic spikes. It is proportional to the firing rate $f$, which means that it dominates in the limit of high firing rates $(f \rightarrow \infty)$ and is negligible in the limit of low firing rates $(f \rightarrow 0)$. The second term, $S_{\mathrm{r}}$, is the contribution of the instantaneous reset of the membrane potential. The last term, $S_{\mathrm{sub}}$, corresponds to the effect of the coupling between the two neurons when the presynaptic neuron is subthreshold. By evaluating these expressions in $\Delta=0$ or $\Delta=\pi$, we can compute $\Gamma^{\prime}(0)$ and $\Gamma^{\prime}(\pi)$ which determine the stability of the in-phase and antiphase states in absence of noise, as 

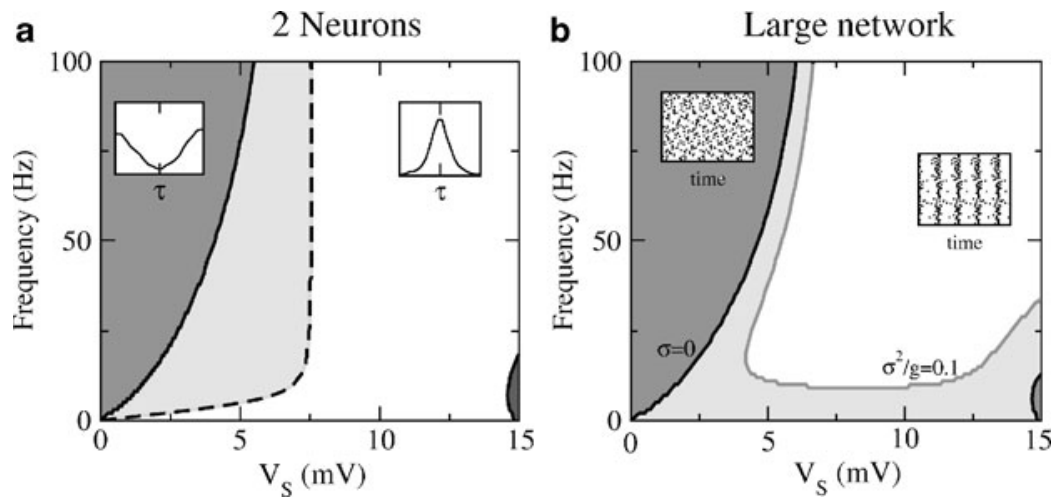

Fig. 15.3 (a), Phase diagram of the stability of the in-phase and antiphase states of a pair of identical QIF neurons coupled by electrical synapses in the weak coupling limit. The solid and dashed boundary lines are defined by the conditions $\Gamma^{\prime}(\pi)=0$ and $\Gamma^{\prime}(0)=0$, respectively (15.58). The inset shows the cross-correlation function $C(\tau)$. In the dark region, the antiphase state is stable and the maximum of $C(\tau)$ is at $\tau=\pi$. In the white region, the in-phase state is stable the maximum of $C(\tau)$ is located at 0 . In the gray intermediate region the stable solution is neither in-phase nor antiphase. A similar phase diagram, but with $V_{\mathrm{r}}$ in the x-axis, is shown in Chap. 14. (b), The phase diagram for the stability of the asynchronous state in a large network of identical QIF neurons all-to-all coupled with electrical synapses in the weak coupling limit in presence of weak noise. The inset shows examples of raster plots. In the dark region, the AS state is stable even for 0 noise. The solid lines are the boundary between the domains in which the asynchronous state is stable and unstable for $\sigma^{2} / g=0$ and $\sigma^{2} / g=0.01$ defined by the condition $\mu_{1}=0$ (15.63). In the white region, the AS state is stable for $\sigma^{2} / g>0.01$ Results are obtained using the QIF model with the time constant $\tau_{0}=10 \mathrm{~ms}$ and the size of the spikes $\theta=1$. The parameter $V_{\mathrm{s}}$ is varied but the reset depth is kept constant: $V_{\mathrm{T}}-V_{\mathrm{r}}=15 \mathrm{mV}$

well as the shape of $\mathrm{CC}$ distributions in presence of noise. A similar expression of the phase interaction function is also given in Chap. 14.

In Fig. 15.3a, we show the phase diagram for the stability of antiphase and inphase locking as a function of $V_{\mathrm{s}}$ (that can be thought as the resting potential) and the firing frequencies. For $V_{\mathrm{s}}<\left(V_{\mathrm{T}}+V_{\mathrm{r}}\right) / 2, Z^{\prime}(\pi)$ is negative (Fig. 15.2) such that $S_{\mathrm{sp}}$ contributes to stabilize the antiphase state for enough small value of $V_{\mathrm{s}}$, and CC displays a peak at $\pi$. Conversely, the always negative value of $S_{\text {sub }}$ for $\Delta=0$ ensures that the in-phase state will be stable for $V_{\mathrm{s}}$ sufficiently large. Between these two regions, the two neurons are locked with a phase between 0 and $\pi$. Moreover, $S_{r}+S_{\text {sub }}$ is positive except for very small or very large values of $V_{\mathrm{s}}$, and $S_{\mathrm{sp}}$ is negligible at low frequency. Therefore, for small firing frequency and large $V_{\mathrm{s}}$, there is a narrow domain of stability of antiphase locking that coincides with a domain of stability of in-phase locking, which means that bistability occurs in the absence of noise and the CC displays two peaks at 0 and $\pi$ in the presence of noise. 


\subsection{Synchrony in a Large Network of QIF Neurons Electrically Coupled}

According to (15.51), the stability of the AS state depends on the Fourier components of the phase interaction function $\Gamma$. For the electrical coupling defined above, these components are given by:

$$
\Gamma_{n}^{\text {gap }}=g Z_{n}\left(v_{-n}+f \theta\right),
$$

where $Z_{n}$, and $v_{n}$ are the nth-Fourier components of the functions $Z(\phi)$ and $v(\phi)$ defined in Eqs. 15.23, 15.20. The variance of the noise, $D$, is given by $D=\sigma^{2}\left(Z^{2}\right)_{0}$ (Kuramoto 1984) [see also (15.28)]. This yields the stability condition for the asynchronous state

$$
\mu_{n}=n g f \theta \operatorname{Im}\left(Z_{n}\right)+n g \operatorname{Im}\left(Z_{n} v_{-n}\right)-n^{2} \sigma^{2}\left(Z^{2}\right)_{0}<0 .
$$

For the asynchronous state to be stable, $\mu_{n}<0$ for all $n$.

The first two terms in (15.63) correspond to the effect of the interaction. The first term represents the effect of the spikes and can be written as

$$
\mu_{n}^{\prime}=-n g f \theta \operatorname{Im}\left(Z_{n}\right)=-\frac{n g f \theta}{2 \pi} \int_{0}^{2 \pi} Z(\phi) \sin (n \phi) \mathrm{d} \phi .
$$

The second term,

$$
\mu_{n}^{\prime \prime}=n g \operatorname{Im}\left(Z_{n} v_{-n}\right)
$$

corresponds to the combined effect of the reset of the membrane potential and its subsequent subthreshold evolution. The sign of the sum of these two terms depends on the parameters, $V_{\mathrm{s}}$ and on the firing frequency, $f$.

The last term in (15.63) corresponds to the effect of the noise. It is always positive. Therefore, as should be expected, noise increases the stability of the asynchronous state (because of the negative sign in front of this term in (15.63)). The stability of the asynchronous state depends on the competition between the first two terms and the last term. In particular, the sign of $\mu_{n}$ depends on the ratio between $\sigma^{2}$ and the coupling strength $g$. Note that because of the factor $n^{2}$ in the last term of (15.63), the stability of the modes increases rapidly with their order.

Note also that $\left(Z^{2}\right)_{0}$ decreases when the firing rate increases. Using Eqs. 15.28 and 15.24, we find that in the limit of large external current $I_{\text {ext }}$ the leading term is given by

$$
\left(Z^{2}\right)_{0} \approx \frac{3 \tau_{0}^{2}}{8 I_{\mathrm{ext}}^{2}}
$$


As in the limit of large firing rate $f$ is proportional to $I_{\text {ext }}$ (see (15.22)), the noise is less efficient at stabilizing the asynchronous state at high firing rates. This effect can be seen in Fig. 15.3b. At large firing rate, the noise affects the stability line much less than at low firing rate.

The qualitative behavior of the quantities $\mu_{1}^{\prime}$ and $\mu_{1}^{\prime \prime}$ is similar to those of $S_{\mathrm{sp}}$ and $S_{\text {sub }}+S_{\mathrm{r}}$, respectively, for a pair of neurons. In particular, $\mu_{1}^{\prime \prime}$ is symmetric around $V_{\mathrm{s}}=\left(V_{\mathrm{T}}+V_{\mathrm{r}}\right) / 2$. Moreover, $\mu_{1}^{\prime}$ increases monotonically from negative values to positive values when $V_{\mathrm{s}}$ increases and it changes sign at $V_{\mathrm{s}}=\left(V_{\mathrm{T}}+V_{\mathrm{r}}\right) / 2$. This is because for $V_{\mathrm{s}}>\left(V_{\mathrm{T}}+V_{\mathrm{r}}\right) / 2$ the function $Z(\phi)$ is skewed toward $\phi<\pi$ (resp. $\phi>\pi)$ where $\sin (\phi)>0$ (resp. $\sin (\phi)<0)$ therefore, for $n=1$, the integral in (15.64) is negative (resp. positive) and $\mu_{1}^{\prime}<0$ (resp. $\mu_{1}^{\prime}>0$ ). In particular, since for $V_{\mathrm{s}}=\left(V_{\mathrm{T}}+V_{\mathrm{r}}\right) / 2, Z(\phi)$ is symmetric around $\phi=\pi$, the integral in (15.64) vanishes for $n=1$ (because $\sin (\phi+\pi)=-\sin (\phi)$ ).

The phase diagram for the stability of the asynchronous state as a function of $V_{\mathrm{s}}$ and the firing frequency is plotted in Fig. 15.3b. It is very similar to the phase diagram for the stability of the antiphase state for a pair of neurons (Fig. 15.3a): asynchronous (vs antisynchronous) state is stable for (1) $V_{\mathrm{s}}$ sufficiently small and frequency sufficiently large or (2) $V_{\mathrm{s}}$ sufficiently large and frequency sufficiently small. The only qualitative difference between these two phase diagrams is associated with the presence of noise. For large enough firing rates, the instability lines for $\sigma^{2} / g=0$ and $\sigma^{2} / g=0.01$ are very close to each other. The distance between the two lines increases when the frequency decreases. At some value, $f=f_{*}$, the two lines separate completely and continue toward the right of the phase diagram (large $V_{\mathrm{s}}$ ). In particular, for $\sigma^{2} / g=0.01$ the asynchronous state is stable for small enough firing rate regardless the value of $V_{\mathrm{s}}$. As a matter of fact, one can show analytically that the asynchronous state is always stable if the firing rate is smaller than a critical value, $f_{*}\left(\sigma^{2} / g\right)$, which vanishes with $\sigma^{2} / g$ and increases with the noise level.

There are two noticeable regimes where the change of synchrony properties is nonmonotonic:

1. In some intermediate range of firing rates, the stability of the asynchronous state varies nonmonotonically with $V_{\mathrm{s}}$ : when $V_{\mathrm{s}}$ increases from 0 , the asynchronous state is first stable, then unstable, and stable again for large $V_{\mathrm{s}}$. The size of the intermediate region in which the asynchronous state is unstable decreases when the noise level increases.

2. If the noise is not too strong, the stability of the asynchronous state varies nonmonotonically in some intermediate range of $V_{\mathrm{s}}<\left(V_{\mathrm{T}}+V_{\mathrm{r}}\right) / 2$. In this domain, the asynchronous state is stable at low firing rates, loses stability when the rate increases but becomes stable again at high firing rates. 


\subsection{Synchrony in Networks of Conductance-Based Neurons Electrically Coupled}

In previous subsections, we have shown analytically how the shape of the iPRC, which depends on the QIF neuronal model parameter $V_{\mathrm{s}}$ and firing frequency, determines the stability of antisynchronous (for two neurons) and asynchronous states in networks of QIF neurons electrically coupled. These results provide a unified framework explaining how synchronization properties depend on detailed intrinsic neuronal properties of conductance-based neurons (models described in Appendix 1). We therefore simulate the dynamics of networks of two or a large number of conductance-based neurons, measure the synchronization properties and interpret the synchronization behavior in term of the shape of the iPRC. The quantities that measure synchrony in the case of two or large number of neurons are defined in the Appendix 2.

\subsubsection{Synchrony via Electrical Synapses Depends on the Intrinsic Conductances of the Neurons}

Our analysis of the QIF model suggests that more generally synchrony may be predicted from the firing frequency and the shape of the iPRC independently of the details of the neuronal dynamics. In particular, neurons coupled with electrical synapses will be more easily synchronized if their iPRC are skewed to the right than to the left. We check whether specific intrinsic neuronal properties that shape the iPRC also determine the synchronization properties of a pair of neurons or a large number of neurons coupled with electrical synapses according to this qualitative rule. In Fig. 15.4, we show the effect of specific currents frequently found in neurons both on neuronal synchronization and the shape of the iPRC.

1. Adding a slow potassium current increases the degree of synchrony between a pair of neurons (peak of $\mathrm{CC}$ ) or in a large network (amplitude of the mean potential membrane). The presence of such current gives also rise to a diminution of the iPRC after the spike and to an increase in the value of $Z^{\prime}(\pi)$ (Fig. 15.4b).

2. In presence of a persistent sodium current, the network activity becomes asynchronous and the two neurons are locked in antiphase. Incidentally, such current leads to an increase of the iPRC after the spike, such that the peak is shifted on the left (Fig. 15.4c).

3. Increasing the leak conductance (due for instance to synaptic conductance) enhances the synchrony level in large network or pair of neurons incorporating a persistent sodium current. This is because the shape of the iPRC is skewed to the right (Fig. 15.4d).

One can understand intuitively how potassium and sodium currents shape the iPRC. The current, $I_{\mathrm{K}}$, increases the refractoriness of the neuron; therefore, it reduces its responsiveness to small depolarization after a spike. A similar effect, 

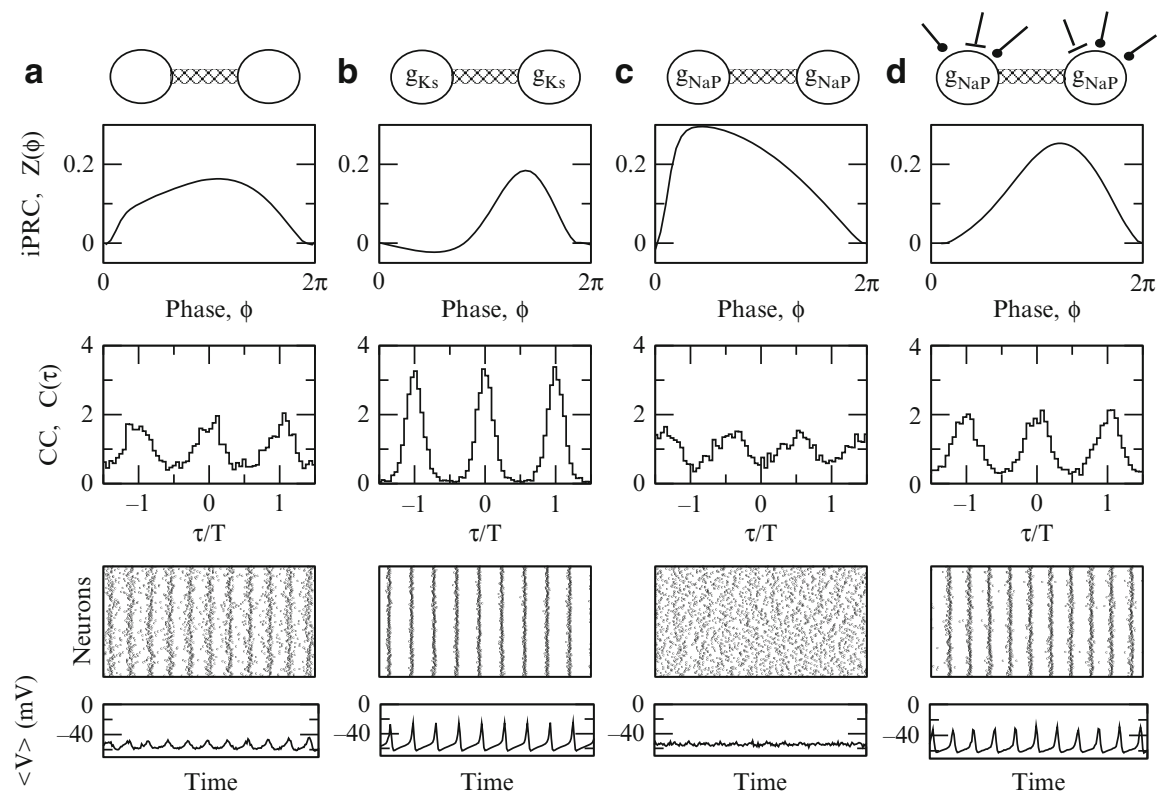

Fig. 15.4 Effect of various intrinsic neuronal conductances on synchronization properties of conductance-based neurons coupled with electrical synapses. Adding various intrinsic conductances to the original conductance-based model (see Appendix 1) modifies the shape of the iPRC (top), and the neuronal synchronization measured by a CC in the case of a pair of neurons (middle), or by a raster-plot and the mean potential in the case of a large network (bottom). Parameters used are $g_{\text {gap }}=0.005 \mathrm{mS} / \mathrm{cm}^{2}, f \approx 50 \mathrm{~Hz}$, and $\sigma=0.3 \mathrm{mV} / \mathrm{ms}^{1 / 2}$. (a), In the original model, the iPRC has a right-side peak and electrical synapses tend to synchronize. (b), Adding a slowpotassium current $\left(g_{K s}=0.4 \mathrm{mS} / \mathrm{cm}^{2}\right)$ decreases the left-side of the iPRC and increases the synchrony level. (c), Adding a persistent sodium current $\left(g_{N a P}=0.4 \mathrm{mS} / \mathrm{cm}^{2}\right)$ shifts the peak of the iPRC to the right and leads to antiphase or asynchronous firing. (d), Increasing the leak conductance in comparison with the model used in (c) shifts the peak of the iPRC to the right and promotes synchronization

but stronger and more lasting, occurs with $I_{\mathrm{Ks}}$. This intuitively explains why we found that larger conductances of these currents skew the iPRC toward the second half of the firing period. Similar effects have been found by Ermentrout et al. (2001) for $\mathrm{M}$ and AHP potassium currents. The current $I_{\mathrm{NaP}}$ is an inward current. It is already activated near rest and depolarizing perturbations amplify this activation. Hence, $I_{\mathrm{NaP}}$ increases the responsiveness of the neuron after a spike and shifts the maximum of the response function toward the first half of the period as we have found here.

In all these examples, changes in intrinsic conductances of neurons affect the synchrony level of electrically coupled neurons, by skewing the iPRC toward the left or the right, similarly to changing $V_{\mathrm{s}}$ in the QIF model. Any other neuronal intrinsic currents that modify the shape of the iPRC may also have impact on synchronization properties, increasing or decreasing it. 
We predict that potassium currents, like $I_{\mathrm{K}}$ and $I_{\mathrm{Ks}}$, tend to promote synchrony of neurons coupled via electrical synapses and that, in contrast, $I_{\mathrm{NaP}}$ tends to oppose it. Our other predictions concern the dependency of the synchrony level with the firing rate. We expect that these conclusions do not depend on the particular models that we have chosen for the intrinsic currents.

\section{Interplay Between Electrical and Chemical Inhibitory Synapses}

Electrical synapses are frequently found to connect GABAergic interneurons in the central nervous system. Therefore, certain types of neurons (e.g., FS cells Gibson et al. 1999; Galarreta and Hestrin 2001 or multipolar bursting Blatow et al. 2003 in neocortex) often interact through both electrical and GABAergic synapses. This raises the question of the effect of their combination in neural synchrony.

\subsection{Neuronal Synchronization with Inhibitory Interactions}

First, we consider the case of inhibitory interactions alone, for which we have chosen a "current-based" model. If the presynaptic neuron fires a spike at time 0 , it injects a current on the postsynaptic neurons equal to $g_{\text {inh }} s(t)$ where

$$
s(t)=\frac{\exp \left(-t / \tau_{1}\right)-\exp \left(-t / \tau_{2}\right)}{\tau_{1}-\tau_{2}}
$$

and $\tau_{1}$ (resp. $\tau_{2}$ ) is the decay (resp. rise) time of the synaptic interaction. If the presynaptic neuron is firing periodically with frequency $f$, the total current can be evaluated by adding up all the spikes. We obtain

$$
s(\phi)=\frac{1}{\tau_{1}-\tau_{2}}\left(\frac{\mathrm{e}^{-\phi /\left(2 \pi f \tau_{1}\right)}}{1-\mathrm{e}^{-1 /\left(2 \pi f \tau_{1}\right)}}-\frac{\mathrm{e}^{-\phi /\left(2 \pi f \tau_{2}\right)}}{1-\mathrm{e}^{-1 /\left(2 \pi f \tau_{2}\right)}}\right)
$$

for $0 \leq \phi<2 \pi$ and $s(\phi)=s(\phi+2 \pi n)$ for any integer number $n$.

The phase interaction function is given by [see (15.27)]:

$$
\Gamma^{\mathrm{inh}}(\phi)=\frac{g_{\text {inh }}}{2 \pi} \int_{0}^{2 \pi} Z(u) s(u-\phi) \mathrm{d} u .
$$

The stability of the asynchronous state is determined by the condition:

$$
-\operatorname{Im}\left(\Gamma_{n}^{\mathrm{inh}}\right)-n \sigma^{2}<0 .
$$



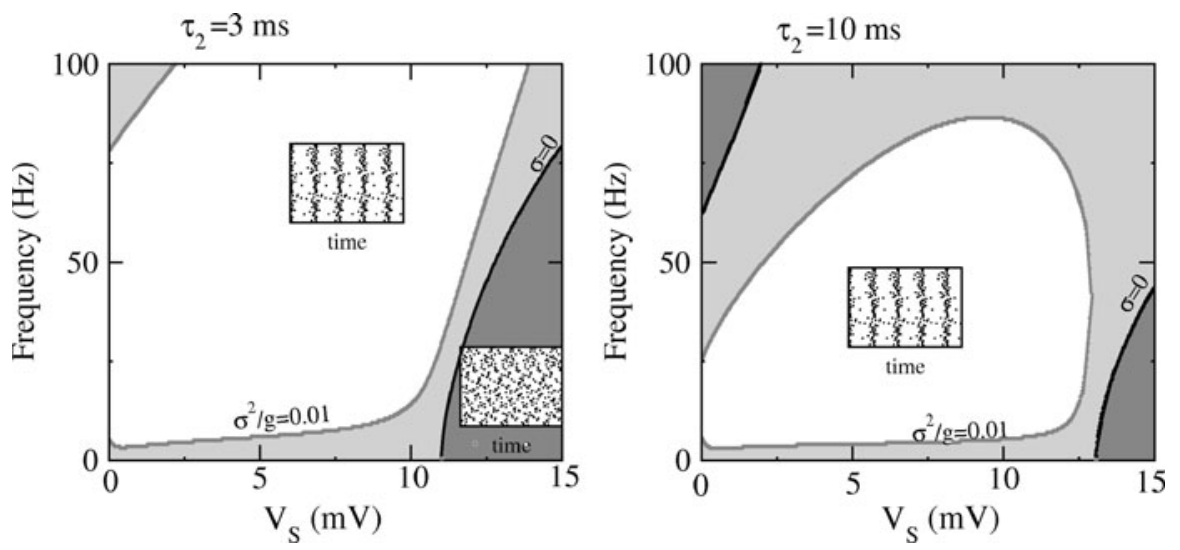

Fig. 15.5 The phase diagram for the stability of the asynchronous state in a large network of identical QIF neurons all-to-all coupled with inhibitory synapses in the weak coupling limit in presence of weak noise. The solid lines are the boundary between the domains in which the asynchronous state is stable and unstable for $\sigma^{2} / g=0$ and $\sigma^{2} / g=0.01$ defined by the condition $\Gamma_{1}=0$. Results are obtained using the QIF model with the time constant $\tau_{0}=10 \mathrm{~ms}$ and synaptic rise time constant $\tau_{1}=1 \mathrm{~ms}$. Left and right phase diagrams are associated with two different synaptic decay time constants of $\tau_{2}=3 \mathrm{~ms}$ and $\tau_{2}=10 \mathrm{~ms}$, respectively

Combining (15.69) and (15.68), $\operatorname{Im}\left(\Gamma_{n}^{\mathrm{inh}}\right)$ writes:

$$
\operatorname{Im}\left(\Gamma_{n}^{\mathrm{inh}}\right)=\frac{g_{\text {inh }}}{A}\left[\left(\frac{1}{\tau_{1} \tau_{2}}-n^{2}\right) \operatorname{Im}\left(Z_{n}\right)-n\left(\frac{1}{\tau_{1}}+\frac{1}{\tau_{2}}\right) \operatorname{Re}\left(Z_{n}\right)\right]
$$

with:

$$
A=2 \pi \tau_{1} \tau_{2}\left(\frac{1}{\left(\tau_{1}\right)^{2}}-n^{2}\right)\left(\frac{1}{\left(\tau_{2}\right)^{2}}-n^{2}\right) .
$$

Inhibitory interactions are characterized by significant synaptic time constants that influence their synchronization properties. The interplay between synaptic time constants and intrinsic properties of neurons is shown in Fig. 15.5, which displays the phase diagram for the stability of asynchronous state as a function of $V_{\mathrm{s}}$, the firing frequencies and the decay time of inhibitory synapses $\tau_{2}$. For rapidly decaying synaptic current, the asynchronous state is stable for low-firing frequencies and large values of $V_{\mathrm{s}}$. A slower decay time constant tends to reduce such domain of stability of the asynchronous state, while it stabilizes the asynchronous state for small values of $V_{\mathrm{s}}$ and large firing rates. 


\subsection{Cooperation and Competition Between Electrical and Inhibitory Synapses}

The approach involving weak coupling theory and iPRC can be used to study systems with combined interactions., i.e., chemical inhibitory and electrotonic. Here we study this situation. We analyze the interplay between these two types of interactions in the synchronization of large networks. A similar analysis can be done in the context of synchronization between pairs of neurons (Pfeuty et al. 2005). In the weak coupling case, any number of interactions can be combined additively. For the case of the combinations of electrical and inhibitory couplings, the effective phase interaction function for dual interaction is:

$$
\Gamma^{\text {dual }}=\Gamma^{\mathrm{inh}}+\Gamma^{\mathrm{gap}}
$$

From (15.69), (15.50), and (15.51), it is straightforward to see that in a network with electrical and inhibitory synapses, the stability of asynchronous state is determined by the condition:

$$
\operatorname{Re}\left(\lambda_{n}^{\text {dual }}\right)=-\operatorname{Im}\left(\Gamma_{n}^{\mathrm{gap}}\right)-\operatorname{Im}\left(\Gamma_{n}^{\mathrm{inh}}\right)-n \sigma^{2}<0
$$

where $\operatorname{Im}\left(\Gamma_{n}^{\mathrm{gap}}\right)$ and $\operatorname{Im}\left(\Gamma_{n}^{\mathrm{inh}}\right)$ are evaluated using (15.63) and (15.71).

From the phase diagram shown in Figs. 15.3 and 15.5 and from (15.74), we can predict synchronization properties of combined interaction from those of each interaction separately. Specifically, distinct domains of parameters (such as $f$, $V_{\mathrm{s}}, \tau_{1}, \tau_{2}$ ) for which the two interactions have opposite or similar effects in synchrony, would define cooperative and competitive regimes of synaptic interplay in synchronization. Figure 15.6 displays these different regimes in the case of a network of QIF neurons.

When $V_{\mathrm{s}}$ is sufficiently small and the frequency sufficiently large, the electrical and inhibitory synapses have opposite effects: electrical synapses reduce the synchronization induced by inhibitory synapses (region C1 of the Figs. 15.6a and the example in Fig. 15.6b). When $V_{\mathrm{s}}$ is sufficiently large and the frequency sufficiently small, the electrical and inhibitory synapses have also opposite effects: inhibitory synapses reduce the synchronization induced by electrical synapses (region C2 of Fig. 15.6a and the example in Fig. 15.6b). Finally for an intermediary region, the two types of synapses cooperate because each type promote synchronization induced by the other type (region C3 in Fig. 15.6a and the example in Fig. 15.6b).

In the cooperative regime, electrical and inhibitory synapses combine their effect to reinforce the robustness of synchrony. When electrical and inhibitory synapses both promote synchrony, this means that the asynchronous state becomes unstable below a given amount of noise $\sigma_{\mathrm{c}}$ such that $\lambda\left(\sigma_{\mathrm{c}}\right)=0$. From (15.74), we can write that $\sigma_{\mathrm{c}}^{\text {gap }}=\sqrt{-\operatorname{Im}\left(\Gamma_{n}^{\text {gap }}\right) / n}$ and $\sigma_{\mathrm{c}}^{\text {inh }}=\sqrt{-\operatorname{Im}\left(\Gamma_{n}^{\text {inh }}\right) / n}$. When both synapses are present, the robustness (defined as the maximal amount of noise compatible with a totally or partially synchronous state) is given by $\sigma_{\mathrm{c}}^{\text {dual }}$. Figure 15.7 shows 

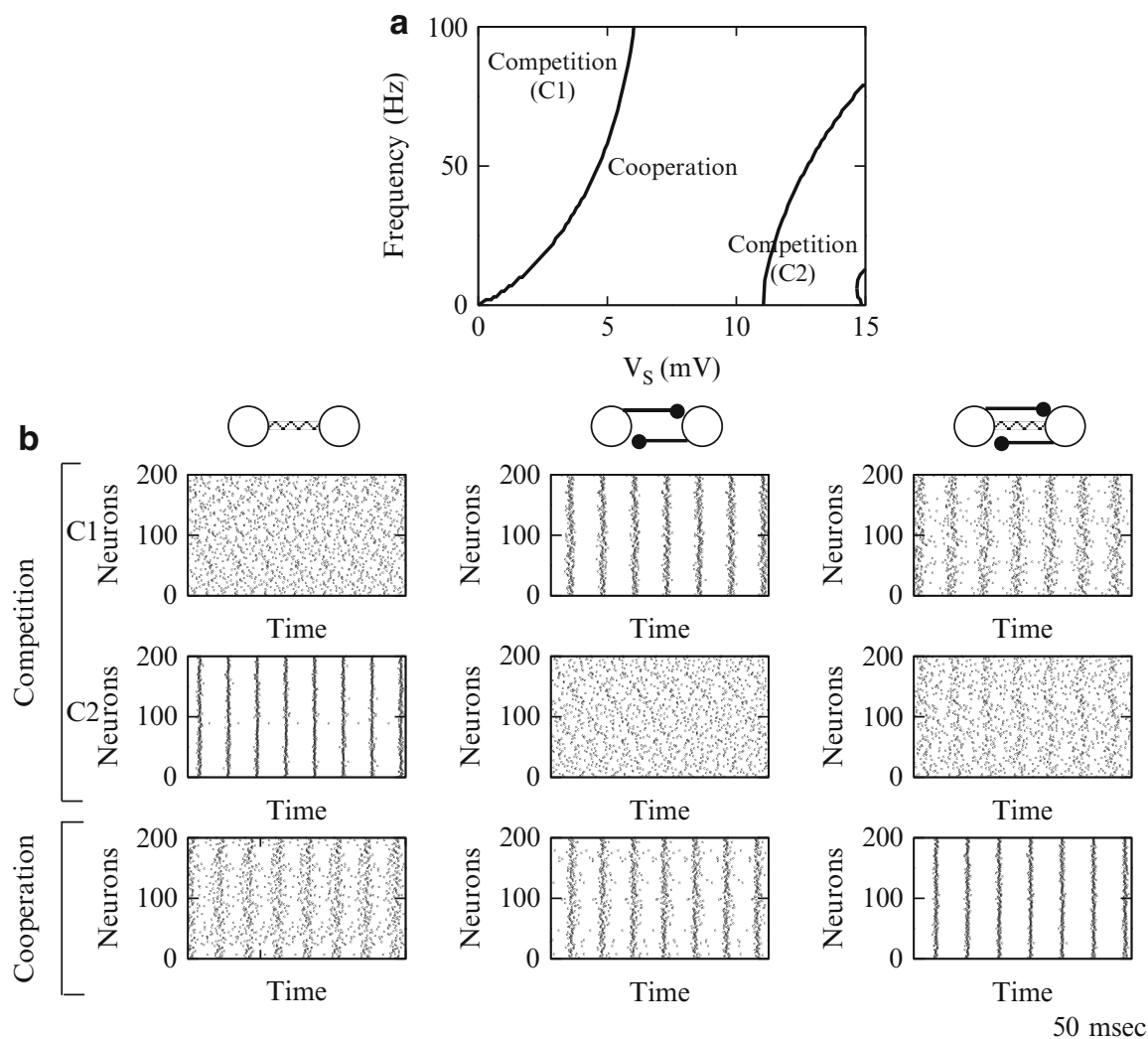

Fig. 15.6 Interplay between electrical and inhibitory synapses in the dynamics of network of weakly coupled QIF neurons. Activating and inactivating time associated with inhibitory currents are $\tau_{1}=1 \mathrm{~ms}$ et $\tau_{2}=3 \mathrm{~ms}$. (a), phase diagram showing three domains of synaptic interplay: In $C_{1}$ and $C_{2}$ regions, electrical and inhibitory synapses have competitive effect in synchronization, one promoting it and the other destroying it. Between these regions, both types of synapses cooperate to promote synchronization. (b), Raster-plots obtained from numerical simulation of large network of QIF neurons in these cooperative and competitive regimes

the respective values of $\sigma_{\mathrm{c}}^{\text {dual }}, \sigma_{\mathrm{c}}^{\text {gap }}$ and $\sigma_{\mathrm{c}}^{\text {inh }}$ as a function of $V_{\mathrm{s}}$. For intermediate values of $V_{\mathrm{s}}$ for which both interactions cooperate to promote synchronization, the robustness $\sigma_{\mathrm{c}}$ increases compared to each interaction alone. In contrast, for extreme values of $V_{\mathrm{s}}$ for which both interactions compete, the robustness decreases. These results obtained with QIF model predict that these cooperative and competitive regimes and their robustness properties also arise in network of conductance-based neurons depending on the frequency, properties of intrinsic currents, and synaptic characteristics. 


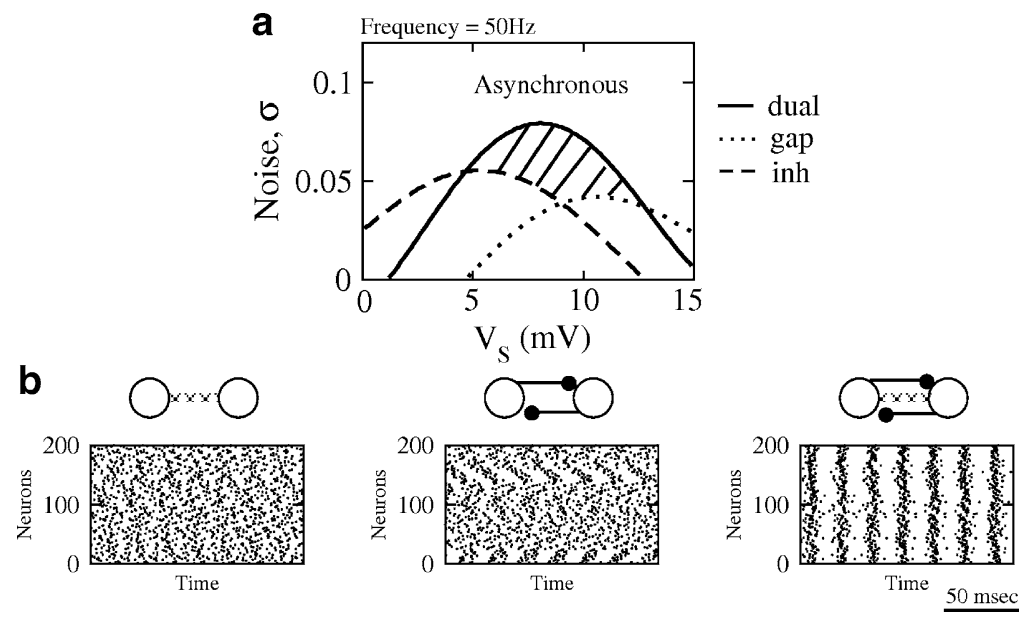

Fig. 15.7 Cooperation and competition regimes between electrical and inhibitory synapses promoting the robustness of synchronization of weakly coupled QIF neurons. (a), Phase diagram displaying the effect of noise on the stability of the asynchronous state. For intermediate value of $V_{\mathrm{s}}$, synchrony is more robust to noise in presence of two interactions. (b), Raster plots displaying an example where combining the two types of synapses compensate the desynchronizing effect of noise (hatched domain)

\subsection{Beyond Weak Coupling: Extension of the Cooperative Regime}

Beyond the weak coupling regime, (15.74) cannot predict anymore the synchronization properties of small or large numbers of neurons. This is the case for each kind of synaptic interaction alone, but especially when the two types of coupling are combined. This can be shown in both networks of interacting QIF neurons or networks of interacting conductance-based neurons.

Figure 15.8 shows the case of a network of conductance-based neurons that incorporate a persistent sodium current. The electrical synapses oppose the synchronization induced by inhibitory synapses in agreement with the prediction of weak coupling theory (Fig. 15.8a). However, electrical synapses, when combined with sufficiently strong inhibitory synapses, favor synchronization (Fig. 15.8b). The amplitude of this synergetic effect depends on the strength of electrical synapses (Fig. 15.8c). Such synergetic cooperation between electrical synapses and strong enough inhibitory synapses contributes to reinforce robustness of synchronized states in the face of different types of noise: (1) the noise in external current, (2) the heterogeneity of external current, (3) the random connectivity (Fig. 15.9). Figure 15.9a shows that the noise in external current reduces synchronization and stabilizes the asynchronous state for a critical value $\sigma_{\mathrm{c}}=0.4 \mathrm{mV} / \mathrm{ms}^{1 / 2}$ without electrical synapses and $\sigma_{\mathrm{c}}=0.8 \mathrm{mV} / \mathrm{ms}^{1 / 2}$ in presence of electrical synapses. Similarly, the presence of electrical synapses increases the robustness to 
a
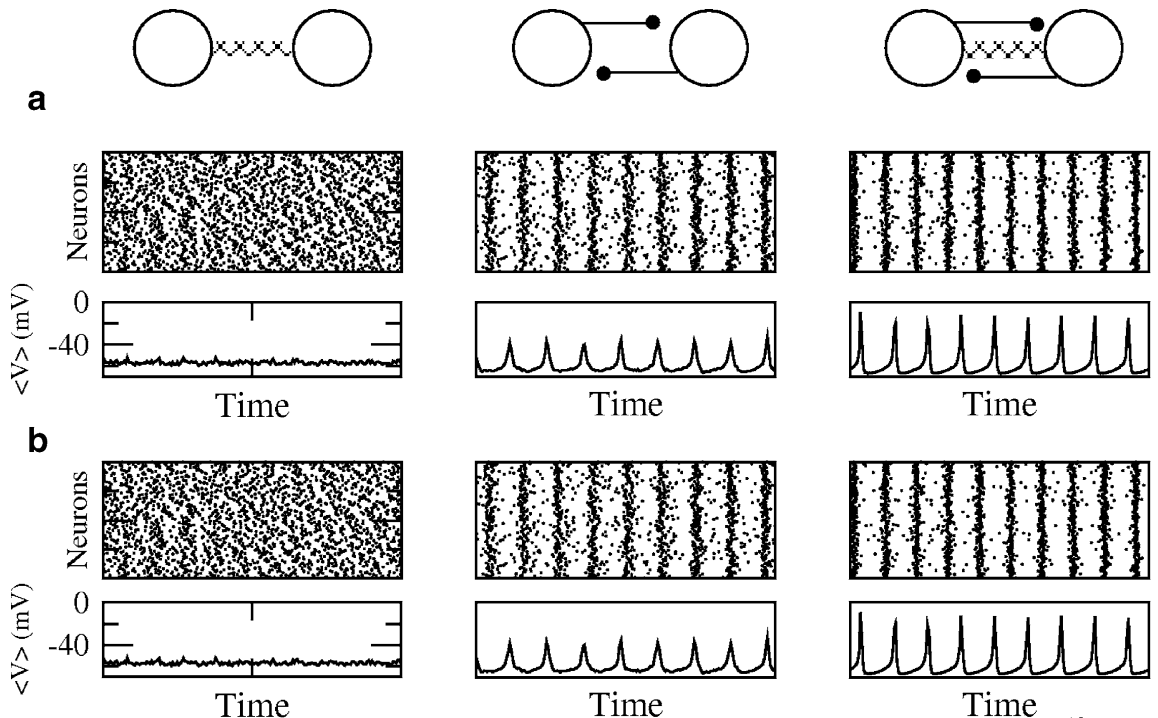

Time

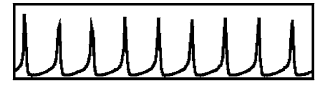

Time
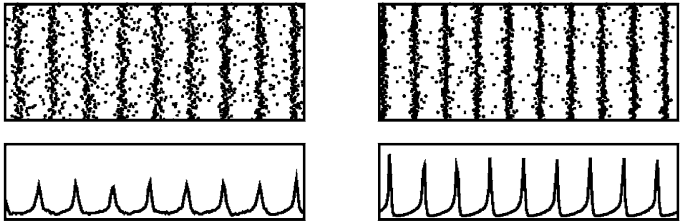

Time

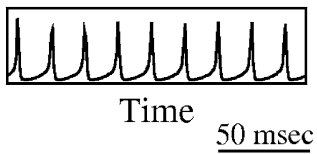

C

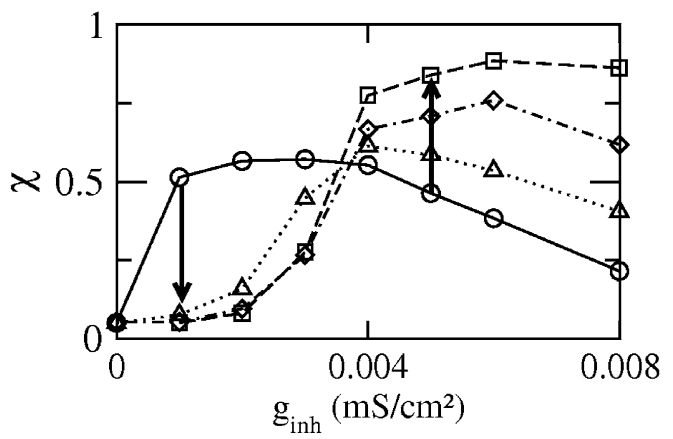

Fig. 15.8 Synergetic cooperation between electrical and inhibitory synapses beyond the regime of weak coupling. Simulations of conductance-based models $\left(g_{\mathrm{NaP}}=0.4 \mathrm{mS} / \mathrm{cm}^{2}, f \approx 50 \mathrm{~Hz}\right.$, $\left.\sigma=0.1 \mathrm{mV} / \mathrm{ms}^{1 / 2}\right) .(\mathbf{a})$, In large networks, weak electrical synapses $\left(g_{\text {gap }}=0.001 \mathrm{mS} / \mathrm{cm}^{2}\right)$ that stabilize the asynchronous state decrease the synchrony induced by weak inhibitory synapses $\left(g_{\text {inh }}=0.001 \mathrm{mS} / \mathrm{cm}^{2}\right)$. (b), Stronger electrical synapses $\left(g_{\text {gap }}=0.004 \mathrm{mS} / \mathrm{cm}^{2}\right)$, although stabilizing the asynchronous state when alone, increase the synchronization induced by strong inhibitory synapses $\left(g_{\text {inh }}=0.005 \mathrm{mS} / \mathrm{cm}^{2}\right)$. (c), Synchrony measure $\chi$ as function of $g_{\text {gap }}$ and $g_{\text {inh. }}$. The synergetic effect requires sufficiently strong inhibitory synapses $\left(g_{\text {inh }}>\right.$ $0.003 \mathrm{mS} / \mathrm{cm}^{2}$ ) while its intensity depends on the strength of electrical coupling (circles: $g_{\text {gap }}=0$; triangles: $g_{\text {gap }}=0.002 \mathrm{mS} / \mathrm{cm}^{2}$; diamonds: $g_{\text {gap }}=0.005 \mathrm{mS} / \mathrm{cm}^{2}$; squares: $g_{\text {gap }}=$ $0.01 \mathrm{mS} / \mathrm{cm}^{2}$ )

heterogeneity or spatial fluctuations in connectivity as shown in Fig. 15.9b,c. For the sake of argument, we have shown an instance of synergetic cooperation in the case where electrical synapses alone are desynchronizing; however, such synergetic effect still occurs when electrical synapses alone are synchronizing. 

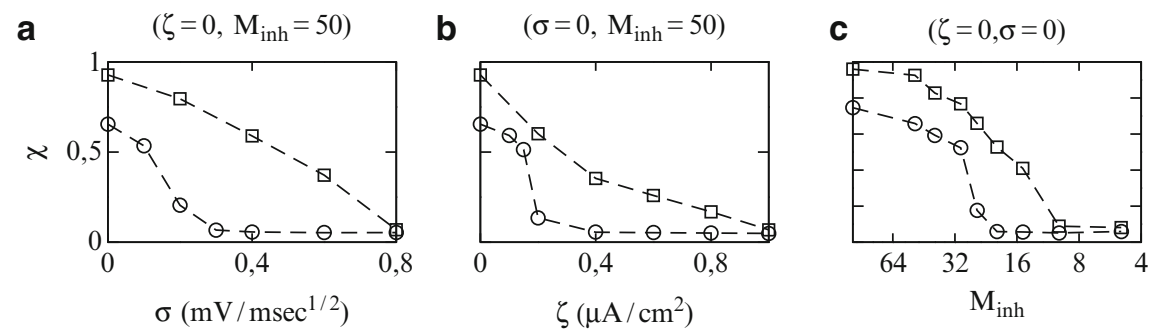

$$
\begin{aligned}
\circ \mathrm{g}_{\text {inh }} & =0.005 \mathrm{mS} / \mathrm{cm}^{2}, \mathrm{~g}_{\text {gap }}=0 \\
\square \mathrm{g}_{\text {inh }} & =0.005 \mathrm{mS} / \mathrm{cm}^{2}, \mathrm{~g}_{\text {gap }}=0.01 \mathrm{mS} / \mathrm{cm}^{2}
\end{aligned}
$$

Fig. 15.9 Effect of synergetic synaptic cooperation on synchronization robustness. Simulations of conductance-based models with a persistent sodium current $\left(g_{N a P}=0.4 \mathrm{mS} / \mathrm{cm}^{2}, f \approx 50 \mathrm{~Hz}\right)$. The synchrony measure $\chi$ is compared between networks coupled with inhibitory synapses $\left(g_{\text {inh }}=\right.$ $\left.0.005 \mathrm{mS} / \mathrm{cm}^{2}\right)$ without (circles) or with (squares) electrical synapses $\left(g_{\text {gap }}=0.01 \mathrm{mS} / \mathrm{cm}^{2}\right)$ as function of the noise, $\sigma(\mathbf{a})$, the heterogeneity $\zeta(\mathbf{b})$, and the mean number of inhibitory synapses $M_{\text {inh }}(\mathbf{c})$

\section{Discussion}

\subsection{Role of Intrinsic Neuronal Properties in Synchrony Mediated by Electrical Synapses}

The dynamics of networks of neurons interacting via chemical synapses have been extensively studied (see e.g., Golomb et al. 2001 and references therein). However, much less theoretical studies have addressed the dynamics of networks in which neurons are coupled by electrical synapses. Previous modeling studies have shown that a pair of electrically coupled neurons may fire in synchrony or in antisynchrony depending on the model properties or on the firing rate (Sherman and Rinzel 1992; Han et al. 1995; Chow and Kopell 2000; Lewis and Rinzel 2003; Cymbalyuk et al. 1994; Alvarez et al. 2002; Saraga et al. 2006). This chapter provides a general framework for understanding this diversity of synchronization behaviors.

We have shown that the synchronization of neurons interacting via electrical synapses can be predicted if one knows the shape of their iPRC. Right-skewed iPRC promotes synchronization while left-skewed iPRC impedes it. In the case of the QIF model, the shape of the iPRC mainly depends on the parameter $V_{\mathrm{s}}$ and the time course of the potential. The effects of $V_{\mathrm{r}}$ and $V_{\mathrm{T}}$ can be studied in a similar way. In conductance-based models as in real neurons, ionic channels and morphology determine the iPRC (Crook et al. 1998; Ermentrout et al. 2001; Oprisan 
and Canavier 2002; Pfeuty et al. 2003). We have focused here on the effect of $I_{\mathrm{NaP}}$ which shifts the maximum of the iPRC toward the left. Let us note that in Chap. 14, the effect of a passive dendritic tree (modeled as two compartments) is also analyzed and it is found that it also shifts the peak of the iPRC to the left. This effect was also found in Pfeuty et al. (2007) for dendritic trees with one compartment. Other inward currents, such as calcium currents, have the same effects on the iPRC and may affect synchrony in a similar way. Potassium currents, in contrast, skew the iPRC to the right (Ermentrout et al. 2001; Pfeuty et al. 2003). Therefore, we predict that potassium and sodium currents have mirror effects on synchrony (Pfeuty et al. 2003). We have verified these predictions in simulations of conductance-based models.

Electrical synapses located on the dendritic compartment of the conductancebased neuron are less synchronizing than if they are located on the soma. One contribution to this effect is the additional delay induced by the dendritic compartment which is like shifting the iPRC to the left. One can similarly predict the effect of the synaptic delays in the inhibitory interactions. Although we did not include these delays in our model, our approach can be straightforwardly extended to take them into account. For given cellular properties this would amount to shifting the iPRC of the neurons to the left. Therefore, sufficiently large delays with respect to the firing period have the same effect as increasing $g_{N a P}$ without delays.

Phase locking of neurons connected by electrical synapses has been also investigated analytically in Chow and Kopell (2000) and Lewis and Rinzel (2003). Both studies found stable antiphase locking at low firing rates and destabilization for large enough firing rates. This can be understood as follows. For a passive integrator, $Z(\phi)=1 /\left[I_{\text {ext }} T\right] \exp \left(\phi T / 2 \pi \tau_{\mathrm{m}}\right)$ where $T$ is the period, $I_{\text {ext }}$ the external current and $\tau_{\mathrm{m}}$, the neuronal membrane time constant (Kuramoto 1984). The value of $Z$ diverges exponentially with the period at all $\phi$ and so do $S_{\text {sub }}$ and $S_{\mathrm{r}}$ [(15.61) and (15.60)]. Detailed analysis reveals that the most divergent of these terms is $S_{\text {sub }}<0$. Hence, for low enough firing rates $\Gamma^{\prime}(\pi)<0$. The term $S_{\mathrm{sp}}$ is positive $\left(Z^{\prime}(\phi)>0\right)$ and decreases with $T$, whereas $S_{\text {sub }}$ and $S_{\mathrm{r}}$ increase. Hence, for a large enough frequency, $\Gamma^{\prime}(\pi)$ changes sign. Therefore, for passive integrators antiphase locking is stable at low firing rates and loses stability as the firing rate increases. In contrast, we have found that the QIF model behaves differently than the LIF except in the limit of very large $V_{\mathrm{s}}$. Therefore, our results show that the predictions of Chow and Kopell (2000) and Lewis and Rinzel (2003) may be relevant only for neurons with strong potassium currents.

\subsection{Validity of the Weak Coupling Approximation}

The stability of the asynchronous state of a large network of all-to-all connected QIF neurons can be calculated analytically in the absence of noise for any coupling strength using the population density method (Abbott and van Vreeeswijk 1999; van Vreeswijk 1996; Hansel and Mato 2001, 2003). An alternative approach is 
the phase reduction method. Although it is only mathematically justified for weak interactions, it leads to nontrivial results, which remain valid in a reasonable range of interaction strengths. Moreover, it is straightforward to study the effect of noise in this framework. These were the motivations to use the phase reduction method in the framework of which all the analytical results of this paper were derived.

In order to assess the validity of the approximation, it is necessary to perform numerical simulations for different coupling strengths. For instance, in Hansel et al. (1993) the synchronization properties of a system of two chemically coupled HH neurons were analyzed numerically. It was found that the coupling strength could be strong enough to change the firing rate of the coupled system in $30 \%$ with respect to the uncoupled neurons, and still the predictions of the phase models analysis were qualitatively correct.

For gap junctions, numerical simulations in this chapter were performed for the coupling conductance $g=0.005 \mathrm{mS} / \mathrm{cm}^{2}$. This corresponds to coupling coefficients (Amitai et al. 2002) $\approx 5 \%$ and to a spikelet size of about $0.5 \mathrm{mV}$. We have checked that similar results are found for conductances four times larger (data not shown). Therefore, the conclusions of the present work are relevant for electrical synapse conductances in the physiological range (Amitai et al. 2002; Galarreta and Hestrin 2001, 2002).

However, one must keep in mind that electrical synapses, in the limit of strong coupling, are always synchronizing regardless of intrinsic properties of neurons or firing rates. At finite coupling strength, an additional instability of the asynchronous state appears at low firing rates and large $V_{\mathrm{s}}$. It corresponds to the impossibility of controlling low firing rates when the coupling is too strong and the AHP is too small. This is because for small AHP, the recurrent electrical synapses interactions tend to depolarize the neurons on the average and this increases their firing rates. This instability is similar to the rate instability in networks of excitatory neurons (Hansel and Mato 2003).

\subsection{Combining Electrical and Inhibitory Synapses: In and Beyond the Weak Coupling Regime}

In the weak coupling regime, the interplay between electrical and inhibitory synapses gives rise to a straightforward outcome (Lewis and Rinzel 2003; Pfeuty et al. 2005). The synchronizing or desynchronizing effects of each interaction alone, which depends on synaptic and neuronal parameters, add when interactions are combined, such as to define cooperative and competitive regimes. In the cooperative regime, electrical synapses can reinforce the robustness of synchrony induced by inhibitory synapses. In the competitive regime, electrical synapses can destroy the neural synchrony induced by inhibitory synapses rather than promoting it.

However, in the case of the combined interaction, weak coupling approximation is no longer valid for realistic coupling strength. In particular, electrical synapses 
become less versatile when the neurons also interact via inhibition, as they always promote synchrony (Pfeuty et al. 2007). This is because of a modulatory effect of inhibition which "renormalizes" the synchronization properties of electrical synapses. This effect was depicted here in the case of electrical synapses which are desynchronizing because of the intrinsic properties of neurons. We found that this is significant for spike-to-spike synchrony as well as for stochastic synchrony. Similar modulatory effects occur when the electrical synapses are desynchronizing because of their distal position (Pfeuty et al. 2007) on dendrites or because of their too sparse connectivity. In all these cases, sufficiently strong inhibition can switch the electrical synapses from being desynchronizing to synchronizing. It should be noted that inhibition also potentiates the synchronizing effect of electrical synapses which, when alone, are already synchronizing. In this case, the function $\sigma_{\mathrm{c}}\left(g_{\text {gap }}\right)$ increases more rapidly with $g_{\text {gap }}$ when the inhibition is strong than when it is weak (Pfeuty et al. 2007).

\subsection{Physiological Relevance of the Cooperative Interplay Between Electrical and Inhibitory Synapses}

Experimental studies in neocortical and hippocampal preparations have revealed that both electrical and inhibitory synapses are often required to generate synchrony of firing. This has been shown in large ensemble of neurons in vitro (Hormuzdi et al. 2001; Traub et al. 2001; LeBeau et al. 2002; Blatow et al. 2003) as well as in pairs of neurons (Tamas et al. 2000; Szabadics et al. 2001). Our results showing that electrical synapses combined with inhibition improve the robustness of synchrony in a way which, to a large extent, does not depend on their location, is in line with these experimental results.

It has been proposed that fast synchronous rhythmic episodes in the $\gamma$ range $(30-100 \mathrm{~Hz})$ observed in cortex and hippocampus, in-vivo or in vitro, are generated within local networks of GABAergic inhibitory interneurons (Whittington et al. 1995; Wang and Buzsáki 1996; Bartos et al. 2007). Although in $\gamma$ oscillations found in slice preparations, interneurons are likely to fire about one action potential per cycle of the rhythm (Cunningham et al. 2003), recent experiments indicate that in $\gamma$ oscillations observed in-vivo during specific behavioral states, GABAergic interneurons fire in a more sparse and irregular way, with a probability of firing per cycle which is between 0.1 and 0.6 (Csicsvari et al. 1999; Tukker et al. 2007). These two modes of synchrony are reminiscent of the two regimes described above, namely spike-to-spike synchrony and stochastic synchrony.

Acknowledgements This work was supported in part by a NATO PST-CLG (reference 977683), the PICS-CNRS (no. 837), the ACI "Neurosciences integratives et computationnelles" (Ministère de la Recherche, France), project SECyT-ECOS A04B04. We gratefully acknowledge David Golomb for fruitful collaboration and discussions. 


\section{Appendix 1}

\section{A1.1 Conductance-Based Models}

The conductance-based model has two compartments although only one somatic compartment is considered in most situations. The second compartment, which corresponds to the dendrite, is passive. For simplicity, we assume that the two compartments have equal areas.

The membrane potential, $V$, of the neuron follows the equation:

$$
\begin{aligned}
C \frac{\mathrm{d} V}{\mathrm{~d} t} & =-I_{\mathrm{L}}-\sum_{\text {ion }} I_{\text {ion }}+I_{\text {ext }}+I_{\text {noise }}-g_{c}\left(V-V_{d}\right), \\
C \frac{\mathrm{d} V_{d}}{\mathrm{~d} t} & =-I_{\mathrm{Ld}}-g_{c}\left(V_{d}-V\right),
\end{aligned}
$$

where $I_{\mathrm{L}}=g_{L}\left(V-V_{L}\right)$ and $I_{\mathrm{Ld}}=g_{L d}\left(V_{d}-V_{L d}\right)$ are leak currents and $\sum_{\text {ion }} I_{\text {ion }}$ is the sum of all voltage dependent ionic currents. The currents $I_{\text {ext }}$ and $I_{\text {noise }}$ are a constant external current and a Gaussian white noise with a zero mean and standard deviation (SD), $\sigma$, respectively.

Our neuron model incorporates an inactivating sodium current, $I_{\mathrm{Na}}$, a delayed rectifier potassium current, $I_{\mathrm{K}}$, a slow potassium current, $I_{\mathrm{Ks}}$ (Erisir et al. 2001) and a noninactivating (persistent) sodium current $I_{\mathrm{NaP}}$ (French et al. 1990).

$$
\begin{aligned}
I_{\mathrm{Na}} & =g_{N a} m_{\infty}^{3} h\left(V-V_{N a}\right), \\
I_{\mathrm{K}} & =g_{K} n^{4}\left(V-V_{K}\right), \\
I_{\mathrm{Ks}} & =g_{K s} s^{4}\left(V-V_{K}\right), \\
I_{\mathrm{NaP}} & =g_{N a P} p_{\infty}\left(V-V_{N a}\right) .
\end{aligned}
$$

The kinetics of the gating variable $h, n, s$ are given by:

$$
\frac{\mathrm{d} x}{\mathrm{~d} t}=\alpha_{x}(V)(1-x)-\beta_{x}(V) x
$$

with $x=h, n, s$ and $\alpha_{h}(V)=0.21 \mathrm{e}^{-(V+58) / 20}, \beta_{h}(V)=3 . /\left(1+\mathrm{e}^{-(V+28) / 10}\right)$, $\alpha_{n}(V)=0.03(V+34) /\left(1-\mathrm{e}^{-(V+34) / 10}\right), \beta_{n}(V)=0.375 \mathrm{e}^{-(V+44) / 80}, \alpha_{s}(V)=$ $0.07(44+V) /\left(1-\mathrm{e}^{-(V+44) / 4.6}\right), \beta_{s}(V)=0.008 \mathrm{e}^{(V+44) / 68}$. The activation functions, $m_{\infty}$ and $p_{\infty}$, are given by: $m_{\infty}(V)=\alpha_{m}(V) /\left(\alpha_{m}(V)+\beta_{m}(V)\right)$, where $\alpha_{m}(V)=0.1(V+35) /\left(1-\mathrm{e}^{-(V+35) / 10}\right), \beta_{m}(V)=4 \mathrm{e}^{-(V+60) / 18}$, and $p_{\infty}(V)=1 /\left(1+\mathrm{e}^{-(V+40) / 6}\right)$. 
Throughout this work, the parameters $g_{N a}=35 \mathrm{mS} / \mathrm{cm}^{2}, V_{N a}=55 \mathrm{mV}$, $g_{K}=9 \mathrm{mS} / \mathrm{cm}^{2}, V_{K}=-75 \mathrm{mV}, g_{L}=0.1 \mathrm{mS} / \mathrm{cm}^{2}, V_{L}=-65 \mathrm{mV}$ and $C=1 \mu \mathrm{F} / \mathrm{cm}^{2}$ are kept constant. $g_{K s}=0, g_{N a p}=0$ in the standard WB model.

In our simulations of network of conductance-based neurons, the network size is $N=1,600$ and the average connectivity is $M=10$.

\section{Appendix 2}

\section{A2.1 Synchrony Measure for Two Neurons}

The synchronization between pairs of neurons is usually defined in terms of the CC function. If the membrane potentials $V_{1}(t)$ and $V_{2}(t)$ are known during a period of time $T$, we define

$$
C_{12}(\tau)=<V_{1}(t+\tau) V_{2}(t)>-<V_{1}(t)><V_{2}(t)>,
$$

where the bracket $\langle\cdots\rangle$ denotes a temporal average:

$$
<x(t)>\equiv \frac{1}{T} \int_{0}^{T} x(t) \mathrm{d} t .
$$

In phase synchrony will be indicated by a $\mathrm{CC}$ with a peak near $\tau=0$, while systems in antiphase will have a peak at $\tau \approx \frac{1}{2 f}$, where $f$ is the frequency of the oscillation.

\section{A2.2 Synchrony Measure for Large Networks}

In a large network of neurons, the activity is asynchronous if at any time the number of action potentials fired in the network is the same up to some random fluctuations. This implies that in a large network, the asynchronous state is unique. When this state is unstable, the network activity is necessarily synchronous. In this case, neurons tend to fire preferentially in some windows of time.

We can characterize the degree of synchrony in a population of $N$ neurons by measuring the temporal fluctuations of macroscopic observables, as, for example, the membrane potential averaged over the population (Hansel and Sompolinsky 1992, 1996; Golomb and Rinzel 1993, 1994; Ginzburg and Sompolinsky 1994). The quantity

$$
V(t)=\frac{1}{N} \sum_{i=1}^{N} V_{i}(t)
$$


is evaluated over time and the variance $\sigma_{V}^{2}=\left\langle[V(t)]^{2}\right\rangle_{t}-\left[\langle V(t)\rangle_{t}\right]^{2}$ of its temporal fluctuations is computed, where $\langle\ldots\rangle_{t}$ denotes time averaging, as defined in (15.83). After normalization of $\sigma_{V}$ to the average over the population of the single cell membrane potentials, $\sigma_{V_{i}}^{2}=\left\langle\left[V_{i}(t)\right]^{2}\right\rangle_{t}-\left[\left\langle V_{i}(t)\right\rangle_{t}\right]^{2}$, we define

$$
\chi(N) \equiv \sqrt{\frac{\sigma_{V}^{2}}{\frac{1}{N} \sum_{i=1}^{N} \sigma_{V_{i}}^{2}}} .
$$

This quantity goes from 0 to 1 . The central limit theorem implies that in the limit $N \rightarrow \infty, \chi(N)$ behaves as:

$$
\chi(N)=\chi(\infty)+\frac{a}{\sqrt{N}}+O\left(\frac{1}{N}\right)
$$

where $a>0$ is a constant, and $O(1 / N)$ means a term of order $1 / N$. In particular, $\chi(N)=1$, if the activity of the network is fully synchronized (i.e., $V_{i}(t)=V(t)$ for all $i)$, and $\chi(N)=O(1 / \sqrt{N})$ if the state of the network activity is asynchronous. In the asynchronous state, $\chi(\infty)=0$. More generally, the larger $\chi(\infty)$ the more synchronized the population. Note that this measure of synchrony is sensitive not only to the correlations in the spike timing but also to the correlations in the time course of the membrane potentials in the subthreshold range.

\section{References}

Abbott, L.F. \& van Vreeswijk, C. (1993). Asynchronous states in networks of pulse-coupled oscillators. Phys Rev E 48, 1483-1490.

Alvarez, L.F., Chow, C., van Bockstaele, E.J. \& Williams, J.T. (2002) Frequency-dependent synchrony in locus coeruleus: Role of electrotonic coupling. P Natl Acad Sci USA 99, 4032-4036.

Amitai, Y., Gibson, J.R., Patrick, A., Ho, B., Connors, B.W. \& Golomb, D. (2002) Spatial organization of electrically coupled network of interneurons in neocortex. $J$ Neurosci 22, 4142-4152.

Bartos, M, Vida, I. \& Jonas, P. (2007). Synaptic mechanisms of synchronized gamma oscillations in inhibitory interneuron networks. Nat Rev Neurosci 8, 45-56.

Beierlein, M., Gibson, J.R. \& Connors, B.W. (2000). A network of electrically coupled interneurons drives synchronized inhibition in neocortex. Nat Neurosci 3, 904-910.

Berger, H. (1929) Uber das Elektrenkephalogramm des Menschen. Archiv für Psychiatrie und Nervenkrankheiten 87, 527-570.

Blatow, M., Rozov, A., Katona, I., Hormuzdi, S.G., Meyer, A.H., Whittington, M.A., Caputi, A. \& Monyer, H. (2003). A novel network of multipolar bursting interneurons generates theta frequency oscillations in neocortex. Neuron 38, 805-817.

Bou-Flores, C. \& Berger, A.J. (2001). Gap junctions and inhibitory synapses modulate inspiratory motoneuron synchronization. J Neurophysiol 85, 1543-1551. 
Brown, E., Moehlis, J. \& Holmes, P. (2004). On the phase reduction and response dynamics of neural oscillator populations. Neural Comput 16, 673-715.

Chow, C.C. \& Kopell, N. (2000). Dynamics of spiking neurons with electrical coupling. Neural Comput 12, 1643-1678.

Crook, S.M., Ermentrout, G.B. \& Bower, J.M. (1998). Spike frequency adaptation affects the synchronization properties of networks of cortical oscillators. Neural Comput 10, 837-854.

Csicsvari, J,, Hirase, H., Czurko, A., Mamiya, A. \& Buzsáki, G. (1999). Fast network oscillations in the hippocampal CA1 region of the behaving rat. J Neurosci 19:RC20, 1-4.

Cunningham, M.O., Davies, C.H., Buhl, E.H., Kopell, N. \& Whittington, M.A. (2003). Gamma oscillations induced by kainate receptor activation in the entorhinal cortex in vitro. $J$ Neurosci 23, 9761-9769.

Cymbalyuk, G.S., Nikolaev, E.V. \& Borisyuk, R.M. (1994). In-phase and antiphase selfoscillations in a model of two electrically coupled pacemakers. Biol Cybern 71, 153-160.

Deans, M.R., Gibson, J.R., Sellitto, C., Connors, B.W. \& Paul, D.L. (2001). Synchronous activity of inhibitory networks in neocortex requires electrical synapses containing connexin 36 . Neuron 31, 477-485.

Erisir, A., Lau, D., Rudy, B. \& Leonard, C.S. (2001). Function of specific K(+) channels in sustained high-frequency firing of fast-spiking neocortical interneurons. J Neurophysiol 82, 2476-2489.

Kopell, N. (1988), Toward a theory of modeling central pattern generators. In: Neural control of rhythmic movements in vertebrates. Cohen, A. (ed.). 369-413. New York: Wiley.

Ermentrout, G.B. (1996). Type I membranes, phase resetting curves and synchrony. Neural Comput 8, 979-1001.

Ermentrout, G.B. (2002). Simulating, analyzing, and animating dynamical systems: a guide to Xppaut for researchers and students (software, environments, tools). Philadelphia: SIAM.

Ermentrout, G.B. \& Kopell, N. (1986). Parabolic bursting in an excitable system coupled with a slow oscillation. SIAM J Appl Math 46, 233-253.

Ermentrout, G.B. \& Kopell, N. (1991). Multiple pulse interactions and averaging in systems of coupled neural oscillators, J Math Biol 29, 195-217.

Ermentrout, B., Pascal, M. \& Gutkin, B. (2001). The effect of spike frequency adaptation and negative feedback on the synchronization of neural oscillators. Neural Comput 13,1285-1310.

French, C.R., Sah, P., Buckett, K.J. \& Gage, P.W. (1990). A voltage-dependent persistent sodium current in mammalian hippocampal neurons. J Gen Physiol 95, 1139-1157.

Friedman, D. \& Strowbridge, B.W. (2003). Both electrical and chemical synapses mediate fast network oscillations in the olfactory bulb. J Neurophysiol 89, 2601-2610.

Fukuda, T. \& Kosaka, T. (2000). Gap-junction coupling linking the dendritic network of GABAergic neurons in the hippocampus. J Neurosci 20, 1519-1528.

Furshpan, E.J. \& Potter, D.D. (1959). Transmission at the giant motor synapses of the crayfish. $J$ Physiol 145, 289-325.

Galarreta, M. \& Hestrin, S. (1999). A network of fast spiking cells in the neocortex connected by electrical synapses. Nature 402, 72-75.

Galarreta, M. \& Hestrin, S. (2001). Electrical synapses between GABA-releasing neurons. Nature Neurosci 2, 425-433.

Galarreta, M. \& Hestrin, S. (2002). Electrical and chemical synapses among parvalbumin fast-spiking GABAergic interneurons in adult mouse neocortex. P Natl Acad Sci USA 19, 12438-12443.

Gibson, J.R., Beierlein, M. \& Connors, B. (1999). Two networks of inhibitory neurons electrically coupled. Nature 402, 75-79.

Ginzburg, I. \& Sompolinsky, H. (1994). Theory of correlations in stochastic neuronal networks. Phys Rev E 50, 3171-3191.

Golomb, D., Hansel, D. \& Mato, G. (2001). Theory of synchrony of neuronal activity. In: Handbook of Biological Physics, Vol 4: Neuro-informatics and neural modeling. Gielen, S. \& Moss, M. (eds.). 887-968. Amsterdam: Elsevier Science. 
Golomb, D. \& Rinzel, J. (1993). Dynamics of globally coupled inhibitory neurons with heterogeneity. Phys Rev E 48, 4810-4814.

Golomb, D. \& Rinzel, J. (1994). Clustering in globally coupled inhibitory neurons. Physica D 72, 259-282.

Han, S.K., Kurrer, C. \& Kuramoto, Y. (1995). Dephasing and bursting in coupled neural oscillators. Phys Rev Lett 75, 3190-3193.

Hansel, D. \& Mato, G. (2001). Existence and stability of persistent states in large neuronal networks. Phys Rev Lett 86, 4175-4178.

Hansel, D. \& Mato, G. (2003). Asynchronous states and the Emergence of Synchrony in Large Networks of Interacting Excitatory and Inhibitory Neurons. Neural Comput 15, 1-56.

Hansel, D., Mato, G. \& Meunier, C. (1993). Phase dynamics for weakly coupled Hodgkin-Huxley neurons. Europhys Lett 23, 367-372.

Hansel, D., Mato. G. \& Meunier, C. (1995). Synchrony in excitatory neural networks. Neural Comput 7, 307-337.

Hansel, D. \& Sompolinsky, H. (1992). Synchrony and computation in a chaotic neural network. Phys Rev Lett 68, 718-721.

Hansel, D. \& Sompolinsky, H. (1996). Chaos and synchrony in a model of a hypercolumn in visual cortex. J Comp Neurosci 3, 7-34.

Hodgkin, A.L. \& Huxley, A.F. (1952). A quantitative description of membrane current and application to conductance in excitation nerve. J Physiol-London 117, 500-544.

Hormuzdi, S.G., Pais, I., Lebeau, F.E.N., Towers, S.K., Rozov, A., Buhl, E., Whittington, M.A. \& Monyer, H. (2001). Impaired electrical signaling disrupts gamma frequency oscillations in connexin 36-Deficient Mice. Neuron 31, 487-495.

Kita, H., Kosaka, T. \& Heizmann, C.W. (1990). Parvalbumin-immunoreactive neurons in the rat neostriatum: a light and electron microscopic study. Brain Res 536, 1-15.

Kopell, N. \& Ermentrout, G.B. (2002). Mechanisms of phase-locking and frequency control in pairs of coupled neural oscillators. In: Fiedler B (ed) Handbook of Dynamical Systems 2:5-55. Amsterdam: Elsevier.

Kuramoto, Y. (1984). Chemical Oscillations, Waves and Turbulence. New York: Springer.

Kuramoto, Y. (1991) Collective synchronization of pulse-coupled oscillators and excitable units. Physica D 50, 15-30.

Landisman, C,E,, Long, M.A., Beierlein, M., Deans, M.R., Paul. D.L. \& Connors, B.W. (2002) Electrical synapses in the thalamic reticular nucleus. J Neurosci 22, 1002-1009.

LeBeau, F.E.N., Towers, S., Traub, R.D., Whittington, M.A. \& Buhl, E. (2002). Fast oscillations networks induced by potassium transient in the rat hippocampus in vivo. $J$ Physiol 542, 167-179.

Lewis, T. \& Rinzel, J. (2003). Dynamics of spiking neurons connected by both inhibitory and electrical coupling. J Comput Neurosci 14, 283-309.

Mann-Metzer, P. \& Yarom, Y. (1999). Electrical coupling interacts with intrinsic properties to generate synchronized activity in cerebellar networks of inhibitory interneurons. J Neurosci 19, 3298-3306.

Oprisan, S.A. \& Canavier, C.C. (2002). The influence of limit cycle topology on the phase resetting curve. Neural Comput 14, 1027-1057.

Perez-Velazquez, J.L., Valiente, T.A. \& Carlen, P.L. (1994). Modulation of gap junctional mechanisms during calcium-free induced field burst activity: a possible role for electrotonic coupling in epileptogenesis. J Neurosci 14, 4308-4317.

Pfeuty, B., Mato, G., Golomb, D. \& Hansel, D. (2003). Electrical synapses and synchrony: the role of intrinsic currents. J Neurosci 23, 6280-6294.

Pfeuty, B., Mato, G., Golomb, D. \& Hansel, D. (2005). The combined effect of electrical synapses in synchrony. Neural Comput 17, 633-670

Pfeuty, B., Golomb, D., Mato, G. \& Hansel, D. (2007). Inhibition potentiates the synchronizing action of electrical synapses. Front Comput Neurosci 1, art. 8.

Saraga, F., Ng, L. \& Skinner, F.K. (2006). Distal gap junctions and active dendrites can tune network dynamics. J Neurophysiol 95, 1669-1682. 
Sherman, A. \& Rinzel, J. (1992). Rhythmogenic effects of weak electrotonic coupling in neuronal models. P Natl Acad Sci USA 89, 2471-2474.

Szabadics, J., Lorincz, A. \& Tamas, G. (2001). Beta and gamma frequency synchronization by dendritic gabaergic synapses and gap junctions in a network of cortical interneurons. J Neurosci $21,5824-5831$.

Tamas, G., Buhl, E.H., Lorincz, A. \& Somogyi, P. (2000). Proximally targeted GABAergic synapses and gap-junctions precisely synchronize cortical interneurons. Nature Neurosci 3, 366-371.

Traub, R.D., Kopell, N., Bibbig, A., Buhl, E., Lebeau, F.E.N. \& Whittington, M. (2001). Gap junction between interneuron dendrites can enhance synchrony of gamma oscillations in distributed networks. J Neurosci 21, 9478-9486.

Tukker, J.J., Fuentealba, P., Hartwich, K., Somogyi, P. \& Klausberger, T. (2007). Cell type-specific tuning of hippocampal interneuron firing during gamma oscillations in vivo. $J$ Neurosci 27 , 8184-8289.

van Kampen, N.G. (1981). Stochastic processes in physics and chemistry. Amsterdam: Elsevier.

van Vreeswijk, C., Abbott, L.F. \& Ermentrout, G.B. (1994). When inhibition not excitation synchronizes neural firing. J Comput Neurosci 1, 313-321.

van Vreeswijk, C. (1996). Partial synchronization in populations of pulse-coupled oscillators. Phys Rev E 54, 5522-5537.

Venance, L., Rozov, A., Blatow, M., Burnashev, N., Feldmeyer, D. \& Monyer, H. (2000). Connexin expression in electrically coupled postnatal rat brain neurons. P Natl Acad Sci USA 97, $10260-10265$.

Wang, X.-J. \& Buzsáki, J. (1996). Gamma oscillation by synaptic inhibition in a hippocampal interneuronal network model. J Neurosci 16, 6402-6413.

Watanabe, A. (1958) The interaction of electrical activity among neurons of lobster cardiac ganglion. Jpn J Physiol 8, 305-318.

Whittington, M.A., Traub, R.D. \& Jefferys, J.G.R. (1995). Synchronized oscillations in interneuron networks driven by metabotropic glutamate receptor activation. Nature 373, 612-615. 